\title{
. \\ Programming cell-free biosensors with DNA strand displacement circuits
}

\author{
Jaeyoung K. Jung ${ }^{1,2,3}$, Chloé M. Archuleta ${ }^{1,2,3}$, Khalid K. Alam ${ }^{4}{ }^{4}$ and Julius B. Lucks $\mathbb{D}^{1,2,3,4,5 \bowtie ~}$
}

\begin{abstract}
Cell-free biosensors are powerful platforms for monitoring human and environmental health. Here, we expand their capabilities by interfacing them with toehold-mediated strand displacement circuits, a dynamic DNA nanotechnology that enables molecular computation through programmable interactions between nucleic acid strands. We develop design rules for interfacing a small molecule sensing platform called ROSALIND with toehold-mediated strand displacement to construct hybrid RNA-DNA circuits that allow fine-tuning of reaction kinetics. We use these design rules to build 12 different circuits that implement a range of logic functions (NOT, OR, AND, IMPLY, NOR, NIMPLY, NAND). Finally, we demonstrate a circuit that acts like an analog-to-digital converter to create a series of binary outputs that encode the concentration range of the molecule being detected. We believe this work establishes a pathway to create 'smart' diagnostics that use molecular computations to enhance the speed and utility of biosensors.
\end{abstract}

$\mathrm{C}$ ell-free biosensing is emerging as a low-cost, easy-to-use and field-deployable diagnostic technology platform that can detect a range of chemical compounds related to human and environmental health ${ }^{1,2}$. At their core, these systems consist of two layers: an RNA or protein-based biosensing layer and a reporter construct output layer. By genetically wiring these layers, a signal is generated when the target compound binds to the biosensor and activates reporter expression (Fig. 1). Reactions are assembled by embedding these layers within cell-free systems and freeze-drying them for easy storage, transportation and rehydration with a sample of interest at the point-of-need ${ }^{1,3}$. Using this approach, cell-free biosensors have successfully detected compounds related to human health such as zinc ${ }^{4}$ and quorum sensing molecules from pathogenic bacteria $^{5}$, drugs such as gamma-hydroxybutyrate ${ }^{6}$ and water contaminants such as fluoride ${ }^{1}$, atrazine ${ }^{2}$, antibiotics and heavy metals ${ }^{7}$.

However, existing cell-free biosensors often lack an information processing layer that can manipulate responses from the sensing layer before signal generation (Fig. 1). Such information processing layers are a natural feature of organisms and enable cells to activate stress responses, guide development and make behavioral decisions on the basis of intracellular and extracellular cues ${ }^{8}$. For this reason, genetic information processing layers that implement logic and feedback have been extensively leveraged and engineered in synthetic cellular systems ${ }^{9,10}$. Similarly, we have previously shown that RNA-based circuits can be added to a cell-free biosensors platform called RNA Output Sensors Activated by Ligand INDuction (ROSALIND) to improve their specificity and sensitivity without engineering the protein biosensors ${ }^{7}$. However, these circuits still directly act on either the sensing or the output layer, limiting our ability to improve and expand their function.

Here, we develop a generalizable information processing layer to enhance and expand the function of ROSALIND by leveraging toehold-mediated DNA strand displacement (TMSD)-a computationally powerful DNA nanotechnology that can process molecular information in vitro ${ }^{11}$. In TMSD, single-stranded DNA (ssDNA) inputs exchange strands with double-stranded DNA 'gates' via complementary base pairing interactions to produce ssDNA output strands. By configuring DNA gates into different network architectures, a range of operations can be performed such as signal restoration ${ }^{12}$, signal amplification ${ }^{13}$ and logic computation ${ }^{14,15}$, much like a general chemical computational architecture ${ }^{16}$. The well-characterized thermodynamics of DNA base pairing enable large networks to be built from simple building blocks. In addition, reaction kinetics can be precisely tuned by changing the strength of the 'toeholds'-single-stranded regions within the DNA gates that initiate the strand displacement process ${ }^{17}$. TMSD has led to the development of powerful devices including in vitro oscillators ${ }^{18}$, catalytic amplifiers $^{19}$, autonomous molecular motors ${ }^{20,21}$ and reprogrammable DNA nanostructures ${ }^{22,23}$. Thus, there is a great potential for TMSD-based information processing to improve cell-free biosensors.

Although TMSD circuits have been used to detect nucleic acid targets such as microRNAs ${ }^{24,25}$ and human pathogens ${ }^{26}$, there are currently no general design rules for triggering TMSD circuits with small molecules to enable their use in cell-free biosensors. We therefore sought to create an interface that can convert the binding event of a chemical target to changes in nucleic acid strands that can trigger TMSD cascades. Allosteric transcription factors (aTFs) naturally create this interface by activating transcription of a programmable RNA sequence upon detection of a chemical target. However, there are substantial challenges in combining aTFs and TMSD circuits to function together in situ, such as interference between RNA polymerase (RNAP) and nucleic acid gates ${ }^{27}$, the lack of experimental characterization of RNA-mediated TMSD circuits $^{28}$ and the complexities of RNA folding hindering TMSD circuit function.

Here, we address these challenges by developing design rules to interface the sensing layers of ROSALIND ${ }^{7}$ with TMSD circuits. We first show that the design of a new DNA signal gate can be optimized to enable T7 RNAP-driven in vitro transcription (IVT) and TMSD within the same reaction. Next, we develop the RNA secondary structure design rules to tune the reaction kinetics of TMSD, notably improving the response speed. We also apply this principle

'Department of Chemical and Biological Engineering, Northwestern University, Evanston, IL, USA. ${ }^{2}$ Center for Synthetic Biology, Northwestern University, Evanston, IL, USA. ${ }^{3}$ Center for Water Research, Northwestern University, Evanston, IL, USA. ${ }^{4}$ Stemloop, Inc., Evanston, IL, USA. ${ }^{5}$ nterdisciplinary Biological Sciences Graduate Program, Northwestern University, Evanston, IL, USA. 凶e-mail: jblucks@northwestern.edu 


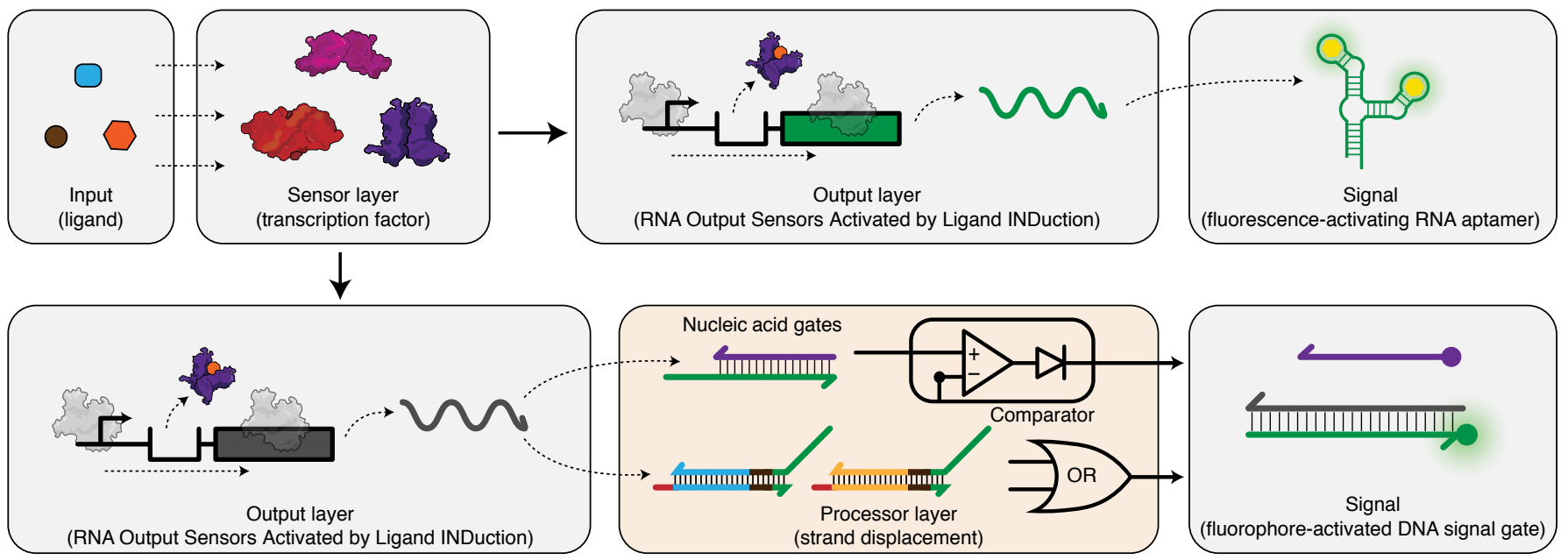

Fig. 1 | Interfacing cell-free biosensors with DNA strand displacement circuits expands and enhances their function. (Upper) A cell-free biosensor typically activates when a target compound (input) binds to a protein transcription factor (sensor layer) that is configured to activate expression of a reporter construct (output layer). This results in the production of a detectable signal such as fluorescence. (Lower) Adding a downstream information processing layer before signal generation can enhance the performance and expand the function of cell-free biosensors by adding computational features such as logic processing and signal comparison. Here, this is implemented by wiring the biosensing output layer to produce a single-stranded RNA capable of activating toehold-mediated strand displacement circuits that generate signal.

to interface TMSD with several different aTFs to create biosensors for their cognate ligands. We then showcase the programmability of the platform by building 12 different circuits that implement seven different logic functions (NOT, OR, AND, NOR, IMPLY, NIMPLY, NAND). Finally, using a model-driven approach, we build a multilayer TMSD circuit that acts like an analog-to-digital converter (ADC) to create a series of binary outputs that encode the concentration range of the target molecule. Taken together, this work demonstrates that TMSD can be used to implement molecular computations to expand the capabilities of cell-free biosensors.

\section{Results}

Engineering TMSD to be compatible with IVT. To interface ROSALIND with TMSD, we first sought to validate that a single-stranded RNA can strand-displace a DNA signal gate. We modified a DNA signal gate from a previous work to create a gate with an eight-nucleotide toehold on its $3^{\prime}$-end, with one strand labeled with a fluorophore and the other strand with a quencher (Supplementary Data 1$)^{29}$. We then designed an invading RNA strand (InvadeR) to be fully complementary to the fluorophore strand so that it strand-displaces the quencher strand to generate a fluorescent output. When we combined purified InvadeR with the DNA signal gate, we observed fluorescence activation over a no InvadeR control (Supplementary Figs. 1 and 2a). In this way, InvadeR behaved similarly to an invading ssDNA strand (InvadeD), although titration of InvadeR resulted in a plateau of fluorescence at lower concentrations than InvadeD (Supplementary Fig. 2a,b). Notably, NUPACK ${ }^{30}$ predicts that InvadeD has a less stable structure than InvadeR, and that InvadeR can bind to itself to form a duplex, which could inhibit the function of InvadeR (Supplementary Fig. 2c-e, Supplementary Data 2 and 3).

We next determined if InvadeR can be transcribed in situ in the presence of the DNA signal gate to generate a signal. Following the ROSALIND platform design, we chose a fast, processive phage polymerase, T7 RNAP, and configured the DNA template to consist of the minimal 17-base pair (bp) T7 promoter sequence followed by two initiating guanines and the InvadeR sequence (Supplementary Data 1). We initially observed that T7 RNAP could generate a fluorescent signal from the DNA signal gate alone (Extended Data
Fig. 1b). On the basis of previous reports ${ }^{27,31-33}$, we hypothesized that T7 RNAP was initiating transcription from the $3^{\prime}$ toehold region of the DNA signal gate, causing strand displacement and signal generation (Extended Data Fig. 1a). To test this hypothesis, we reversed the polarity of the DNA signal gate to include a $5^{\prime}$ toehold end and observed no fluorescence signal from the $5^{\prime}$ toehold DNA signal gate (Extended Data Fig. 1b). Urea-polyacrylamide gel electrophoresis (urea-PAGE) analysis of RNA species from each IVT reaction also showed RNA side products only from the reaction with the $3^{\prime}$ toehold DNA signal gate (Extended Data Fig. 1c). We confirmed that $2^{\prime}-O$-methlyation of the DNA signal gate ${ }^{33}$ similarly eliminates spurious transcription (Extended Data Fig. 1d-f).

Interfacing IVT with TMSD outputs. With the optimized DNA signal gate design, we next used TMSD to track RNA outputs generated by T7 RNAP-driven IVT in situ. We focused on optimizing the design of InvadeR for rapid signal generation. On the basis of the results in Supplementary Fig. 2, we hypothesized that the secondary structure of InvadeR would play a critical role in TMSD efficiency, for example by interfering with toehold binding and strand inva$\operatorname{sion}^{34,35}$. To test this hypothesis, we designed five different variants of InvadeR each with varying predicted stabilities and secondary structures on the $3^{\prime}$-end (Fig. 2a). When an equimolar amount of each gel-purified InvadeR variant was added to the DNA signal gate, we observed that the magnitudes of fluorescence signals were ordered according to the predicted minimum free energies of each variant (Fig. 2b). Furthermore, each strengthened version showed substantially lower fluorescence than the corresponding un-strengthened variant.

We then tested the TMSD reaction kinetics of the variants transcribed in situ. When $50 \mathrm{nM}$ of the DNA template encoding each InvadeR variant was added to the IVT reaction mixture (Fig. $2 \mathrm{c}$ ), we observed the fastest fluorescence activation from variant 3 followed by variants 2 and 1 (Fig. 2 d), in agreement with the previous experiment. We also observed slower responses from the strengthened versions, reaffirming our hypothesis (Fig. 2e,f).

However, we observed discrepancies between the quantitative predicted secondary structure stabilities and the reaction kinetics. Specifically, the strengthened variants with lower minimum 


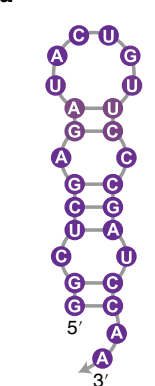

Variant 1

$-5.7 \mathrm{kcal} \mathrm{mol}^{-1}$

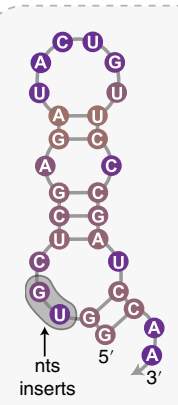

Variant 2

$-3.5 \mathrm{kcal} \mathrm{mol}^{-1}$

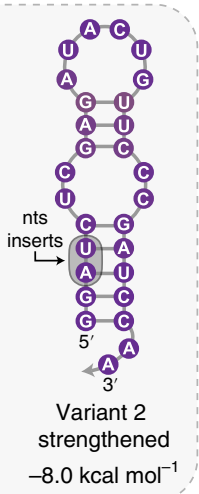

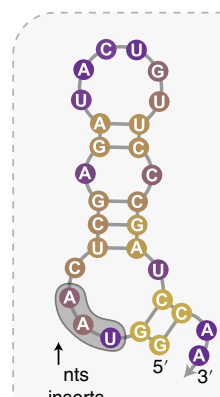

inserts

Variant 3

$-2.8 \mathrm{kcal} \mathrm{mol}^{-1}$

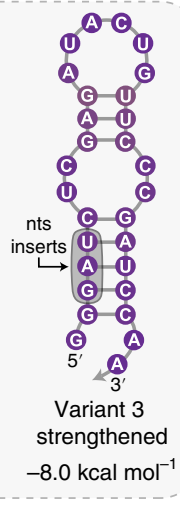

0.2

0

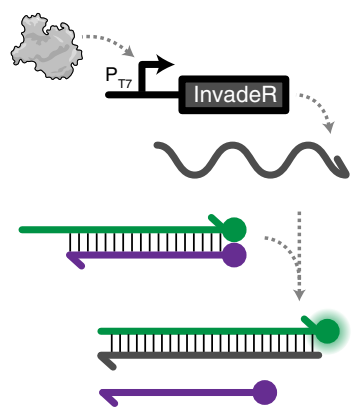

Base pairing probability

d

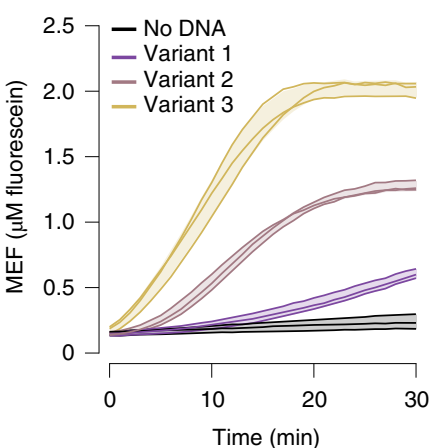

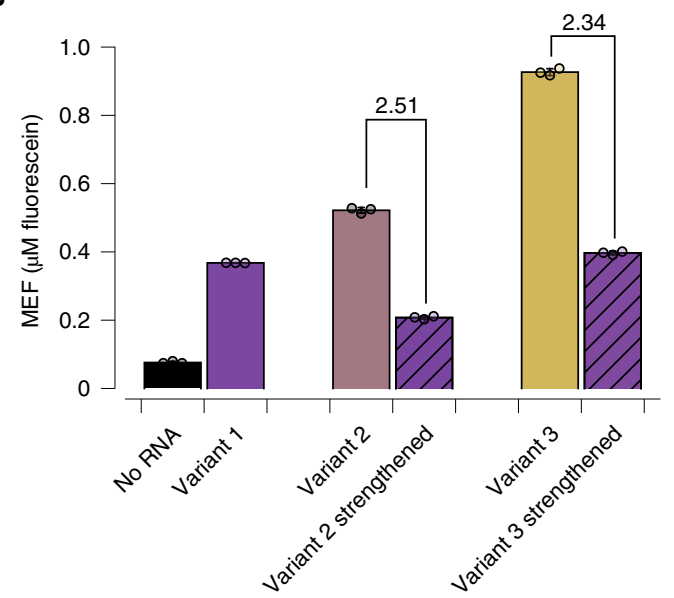

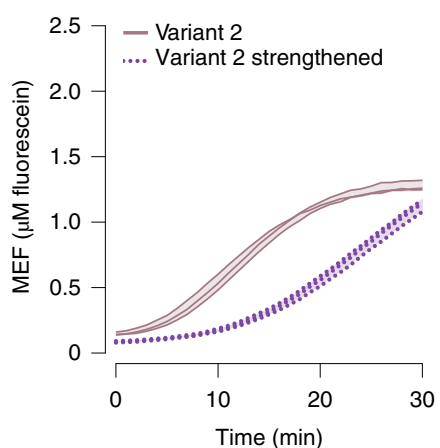

$\mathbf{f}$

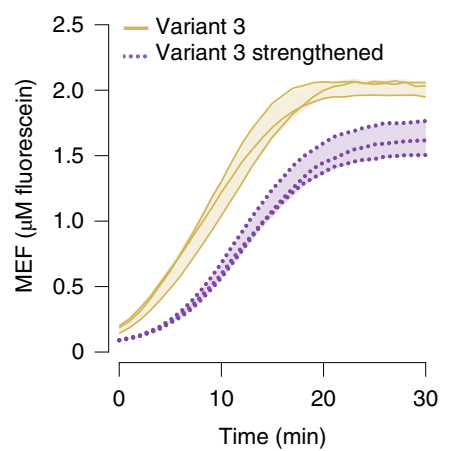

Fig. 2 | Secondary structure of InvadeR impacts TMSD efficiency. a, Three different variants of InvadeR were designed. Variant 1 includes the two initiating guanines followed by the sequence fully complementary to the fluorophore strand. For variants 2 and 3 , two or three additional nucleotides were inserted between the initiating guanines and the InvadeR sequence (shaded regions), such that they disrupt the secondary structure at its base. Strengthened versions of variants 2 and 3 were created to stabilize the structure, while keeping the number and positions of the inserted nucleotides consistent. All variants have the same number of nucleotides that interact with the DNA signal gate. Minimum free energies and base pairing probabilities for each structure are predicted using NUPACK at $37^{\circ} \mathrm{C}^{30}$. nts, nucleotides. b, Gel-purified InvadeR variants ( $5 \mu M$ ) were added to an equimolar amount of the DNA signal gate, and fluorescence activation was quantified. Variant 3 generates the highest fluorescent signal followed by variants 2 and 1 , whereas both strengthening mutants show a decrease in signal from their respective variants by the fold reduction indicated above the bars. c, When a DNA template encoding InvadeR is included with T7 RNAP and the DNA signal gate, the RNA output can be tracked in situ by monitoring fluorescence activation from the signal gate. d, Comparison of fluorescence kinetics of the three variants from IVT using an equimolar DNA template (50 nM) or a no-template negative control. e,f, Comparison of fluorescence kinetics between variants and their strengthening mutants for variant 2 (e) and variant 3 (f), shows that strengthening base pairs negatively impact fluorescence kinetics. All data shown are $n=3$ independent biological replicates each plotted as a point (b) or a line (d-f) with raw fluorescence standardized to MEF ( $\mu$ M fluorescein). Each bar height in $\mathbf{b}$ represents the average over these replicates. Error bars (b) and shading (d-f) indicate the average of the replicates \pm s.d.

free-energy values show faster reaction kinetics and higher end-point fluorescence values than variant 1 (Fig. $2 \mathrm{~d}-\mathrm{f}$ ), conflicting with the results observed in Fig. $2 \mathrm{~b}$. We discovered that varying the T7 RNAP transcription efficiency of each DNA template ${ }^{36}$ contributes to this discrepancy, although RNA secondary structure has a greater impact on the TMSD response speed (Extended Data Fig. 2). We also found that adding a T7 terminator sequence at the end of the DNA template speeds up the reaction, although not considerably (Supplementary Fig. 3 and Supplementary Data 3).

Together, these results show that incorporating both secondary structure and transcription efficiency considerations into InvadeR design principles can be leveraged to enhance reaction speed.

Interfacing cell-free biosensors with TMSD outputs. Next, we determined whether the transcription of InvadeR can be regulated with an aTF to create a cell-free biosensor that uses TMSD outputs. This required us to insert an aTF operator sequence in between the T7 promotor and InvadeR sequence to allow aTF transcription regulation. We previously demonstrated that the spacing between the $\mathrm{T} 7$ promoter sequence and the aTF operator sequence is important for efficient regulation of IVT in ROSALIND reactions ${ }^{7,37}$. Similarly, we found that a 2-bp spacer generates a robust TMSD signal without TetR, which was reduced to nearly baseline levels when regulated by TetR (Fig. 3a,b).

Using the 2-bp spacer, we next determined whether TetR can be de-repressed with its cognate ligand, anhydrotetracycline (aTc) to allow transcription of InvadeR (Fig. 3c). When a range of aTc concentrations was added to IVT reactions each containing $50 \mathrm{nM}$ DNA template, $5 \mu \mathrm{M}$ TetR dimer and $5 \mu \mathrm{M}$ DNA signal gate, we observed a strong repression down to low micromolar amounts of aTc, with half-maximal induction between 2.5 and $5 \mu \mathrm{M}$ of aTc (Fig. 3d).

Owing to the rapid speed of TMSD reactions ${ }^{17}$, we hypothesized that the ligand-mediated induction speed of the InvadeR output would be much faster than the previously used ROSALIND RNA aptamer output that is subject to slow chromophore binding and 

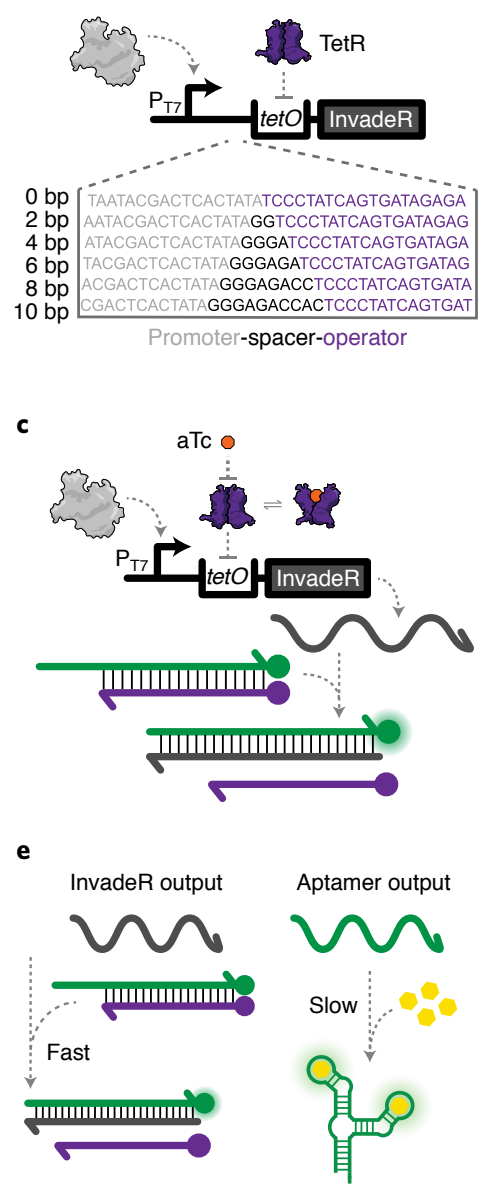

b

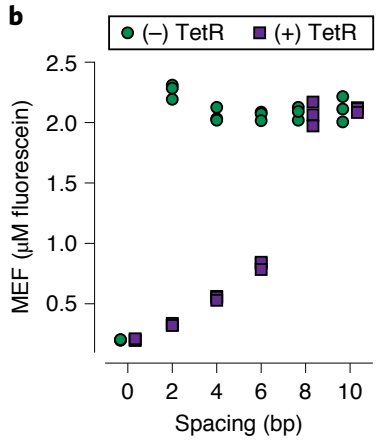

d

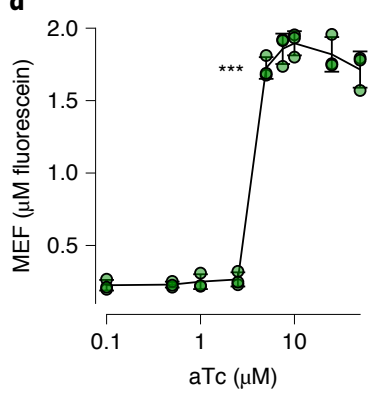

f $\quad(+) 5 \mu \mathrm{M}$ TetR, $10 \mu \mathrm{M}$ aTc

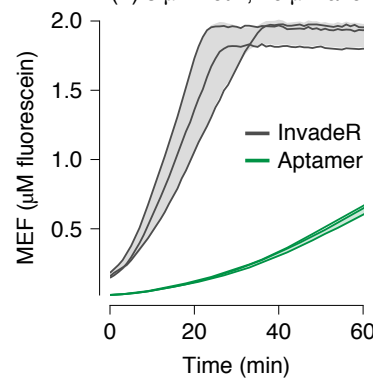

Fig. 3 | Transcription of InvadeR can be regulated by aTFs. a, IVTs can be allosterically regulated with a template configured to bind a purified aTF (TetR) via an operator sequence (tetO) placed downstream of the T7 promoter. A series of spacers at 2-bp intervals was constructed to evaluate the impact of spacer length on the ability of TetR to regulate the transcription of InvadeR. b. Endpoint data (at $1 \mathrm{~h}$ ) shown for promoter-operator spacer variants regulated (with $5 \mu \mathrm{M}$ TetR dimer, 50 nM DNA template) and unregulated (without TetR). c, Induction of a TetR-regulated IVT reaction occurs in the presence of the cognate ligand, aTc, which binds to TetR and prevents its binding to tetO. This allows transcription to proceed, leading to fluorescence activation via TMSD. d, Dose response with aTc, measured at $1 \mathrm{~h}$ with $50 \mathrm{nM}$ DNA template and $5 \mu \mathrm{M}$ TetR dimer. The lowest ligand concentration at which the signal is distinguishable from the background was determined using a two-sided, heteroscedastic Student's $t$-test against the no-ligand condition, and the $P$ value range is indicated by asterisks ( ${ }^{\star \star}{ }^{\star} P<0.001,{ }^{\star \star} P=0.001-0.01,{ }^{\star} P=0.01-0.05$, the exact $P$ value for $5 \mu \mathrm{M}$ aTc $=5.064 \times 10^{-5}$ ). Exact $P$ values along with degrees of freedom for all ligand concentrations tested can be found in the source data. Data for the no-ligand condition were excluded because the $x$ axis is on the log scale and are presented in the source data. e, The speed of the TMSD output is faster than that of the RNA aptamer output. $\mathbf{f}$, Comparison of fluorescence kinetics between the TetR-regulated InvadeR and aptamer outputs when induced with $10 \mu \mathrm{M}$ aTc. All data shown are $n=3$ independent biological replicates each plotted as a point $(\mathbf{b}, \mathbf{d})$ or a line (f) with raw fluorescence values standardized to MEF ( $\mu \mathrm{M}$ fluorescein). Error bars (d) and shading (f) indicate the average of the replicates \pm s.d.

misfolding $34,38,39$ (Fig. 3e). As expected, we observed that the InvadeR platform activates fluorescence visible in $\sim 10 \mathrm{~min}$, which is approximately five times faster than the RNA aptamer platform (Fig. 3f).

Overall, these results demonstrate that an aTF-based biosensor can be successfully interfaced with TMSD outputs, leading to immediate improvements in reaction speed.

Optimizing InvadeR designs for different aTF families. We next determined whether the system is compatible with different families of aTFs to detect various classes of small molecules. In addition to TetR, we chose $\operatorname{TtgR}^{40}$ and $\mathrm{SmtB}^{41}$ as representative aTFs of the MarR family ${ }^{42}$ and SmtB/ArsR family ${ }^{43}$, respectively. We placed the cognate operator sequence of each aTF 2-bp downstream of the T7 promoter and immediately upstream of the InvadeR sequences (Extended Data Fig. 3a-c). When tetracycline was used to induce TetR-regulated reactions, we observed a strong and robust fluorescent signal visible in $\sim 10 \mathrm{~min}$ (Extended Data Fig. 3d,g). Similarly, when naringenin, a cognate ligand of TtgR, was added to TtgR-regulated reactions, we again saw robust fluorescence activation only in the presence of naringenin (Extended Data Fig. 3e,h). However, introducing the smtO sequence resulted in much slower reaction speeds even in unregulated reactions (Extended Data Fig. 4c). Interestingly, the smtO sequence is predicted to form a strong hairpin with the InvadeR sequence (Extended Data Fig. 4a), which on the basis of our results in Fig. 2 could impact reaction speeds. To improve the reaction kinetics, we designed an RNA structural insulation module to prevent the intramolecular smtO:InvadeR folding (Extended Data Fig. 4), which showed faster signal activation of a SmtB-regulated reaction in the presence of $\mathrm{ZnSO}_{4}$ with low background signal (Extended Data Fig. 3f,i).

Together, these results demonstrate that RNA design strategies can be used to modularly extend the ROSALIND platform with TMSD circuits.

Performing logic computation with cascaded TMSD circuits. The interface of cell-free biosensors with TMSD provides opportunities to engineer modular devices that can perform programmed tasks in response to small molecule inputs. This is especially true because TMSD circuits have simpler design rules than protein circuits $^{44}$, there exist computational models that accurately predict their behavior $^{34,35}$ and there are an emerging set of TMSD circuit design tools ${ }^{45,46}$. We therefore leveraged these features to create a TMSD information processing layer for ROSALIND.

We first designed and built logic gates to process two different ligands as inputs to the system. Specifically, we adapted previous designs of TMSD logic gates, AND and OR, that detect nucleic acid inputs $^{12,14,15,46}$ (Fig. 4). We implemented OR logic by designing DNA OR gates that act as an intermediate layer between InvadeR and the signal gate. Once the transcription of InvadeR is triggered by a chemical ligand, InvadeR performs TMSD on its corresponding DNA OR gate to release a ssDNA output strand, which can subsequently invade the DNA signal gate to produce a signal (Fig. 4a and Supplementary Data 4$)^{46}$. The two DNA OR gates share the same output domain (green), but are activated by InvadeR regulated by two different aTFs. Including these OR gates alongside DNA templates, aTFs and the signal gate led to fast signal generation except when no target ligands were present (Fig. $4 \mathrm{~b}$ and Supplementary Data 5). Modeling the OR gate using a set of ordinary differential equations (ODEs) that describe the IVT and TMSD reactions (see Supplementary Information for details on the ODE model used) matched the experimental trends (Extended Data Fig. 5a and Supplementary Data 6).

Next, we designed an RNA-based TMSD AND gate by adapting a previous AND gate design ${ }^{47}$ that consists of three domains: domain 1 complementary to InvadeR 1 (blue), domain 2 complementary to InvadeR 2 (orange) and domain 3 complementary to 
the DNA signal gate (green) (Fig. 4c and Supplementary Data 5). InvadeR strands were designed to have different toehold sequences to minimize unwanted toehold binding to the incorrect AND gate domain. Implementation of this architecture in sensing reactions using TetR and SmtB initially led to no fluorescence activation in any condition (Extended Data Fig. $6 \mathrm{~b}$ ). To drive TMSD reactions forward, we incorporated mismatches in the AND gate that act as TMSD thermodynamic drivers ${ }^{48}$ (Extended Data Fig. 6a). Although the thermodynamic drivers showed signal activation by InvadeR, substantial leak was observed with the InvadeR 1 only condition (Extended Data Fig. 6c). To reduce this leak, we implemented an additional design feature called a clamp in domain 3 of the AND gate which prevents partial TMSD in the presence of InvadeR 1 only ${ }^{49}$. By increasing the clamp length to $7 \mathrm{bp}$, we built an AND gate that requires both tetracycline and $\mathrm{ZnSO}_{4}$ inducible InvadeR strands for signal generation (Fig. 4d, Extended Data Figs. $5 \mathrm{~b}$ and 6 and Supplementary Data 5).

Building more complex logic gates beyond AND and OR requires a NOT circuit, which blocks signal in the presence of a target ligand. To achieve signal inversion, we designed an RNA NOT gate that is capable of sequestering InvadeR away from the DNA signal gate (Fig. 4e and Supplementary Data 4$)^{47,50}$. To bias InvadeR binding to the RNA NOT gate, we included three design features: (1) a longer toehold on the RNA NOT gate than on the DNA signal gate; (2) additional base pairing in the loop of the RNA NOT gate; and (3) a mismatch (highlighted in red) between InvadeR and the DNA signal gate (Extended Data Fig. $6 f-j$ ). We also designed a spacer sequence between the RNA NOT gate and the tet $O$ operator to structurally insulate the tet $O$ sequence from the NOT gate sequence (Fig. 4e). When these design features were implemented, we observed signal reduction in the presence of tetracycline (Fig. 4f, Extended Data Fig. 5c and Supplementary Data 5). No signal inversion was observed from a control template whose sequence is shuffled from the tetracycline-inducible RNA NOT gate (Extended Data Fig. 7a,b). The same design architecture was applied to build a $\mathrm{ZnSO}_{4}$-inducible RNA NOT gate (Extended Data Figs. 5d, 6k-m and $7 \mathrm{c}, \mathrm{d}$, and Supplementary Data 4 and 5).

These results establish a set of design rules for building cascaded TMSD circuits for more complex logic gate computation.

Layering gates to perform complex logic computations. We next layered the basic logic components to perform complex logic computation including NOR, NAND, IMPLY and NIMPLY.

We began with NOR-an inversion of the OR gate that generates signal only when all inputs are absent-by combining two RNA NOT gates each regulated by TetR or SmtB (Fig. 5a and Supplementary Data 4). With both RNA NOT gates designed to sequester the same constitutively expressed InvadeR, we observed fluorescence activation only in the absence of both ligand inputs (Fig. 5b, Extended Data Fig. 5e and Supplementary Data 5).

Next, we focused on the A IMPLY B architecture, which blocks signal only when $\mathrm{A}$ is present and $\mathrm{B}$ is absent. The $\mathrm{ZnSO}_{4}$ IMPLY tetracycline gate was built by layering the tetracycline-induced DNA OR gate with the $\mathrm{ZnSO}_{4}$-induced RNA NOT gate (Fig. $5 \mathrm{c}$ and Supplementary Data 4). When implemented, the gate generated signal in all input conditions except when only $\mathrm{ZnSO}_{4}$ is present (Fig. 5d and Extended Data Fig. 5f). We note, however, that the built-in mismatch between InvadeR and the signal gate (highlighted in red) resulted in a slower and lower fluorescent signal from the no input or both input condition than from the tetracycline only condition, and similar effects were observed from the tetracycline IMPLY $\mathrm{ZnSO}_{4}$ gate (Extended Data Figs. 5g and 7e,f). Interestingly, IMPLY gates can also be built without the DNA OR gate, which allows direct interactions between the ligand-induced InvadeR strand and the signal gate (Extended Data Figs. 5h,i and $7 g-j$ ). The ability to design alternative gate architectures for the same logic computation highlights the platform's modularity.

We then constructed a NAND gate, which combines NOT and AND gates to produce signals in all conditions except when both inputs are present. We explored two design options: (1) inversion of an AND gate output strand (A NAND B=NOT (A AND B)); and (2) two RNA NOT gates integrated into the OR gate architecture $($ NOT $(A$ AND B) $=$ NOT A OR NOT B). Because of the sequence constraints imposed by the first design option, we chose to build the NAND gate using the second option (Fig. 5e). This design involves four different DNA templates-two unregulated templates each encoding InvadeR for its corresponding DNA OR gate and two regulated templates each encoding the RNA NOT gate capable of sequestering its respective InvadeR. To avoid the slower TMSD reaction kinetics observed in the IMPLY gate design, we instead introduced a thermodynamic driver in the RNA NOT gate (highlighted in red) to favor TMSD of InvadeR with the RNA NOT gate over that with the DNA OR gate (Fig. 5e). When implemented, both tetracycline and $\mathrm{ZnSO}_{4}$ were required to prevent signal generation (Fig. 5 f and Extended Data. Fig. 5j).

Finally, we designed the A NIMPLY B gate, which combines AND and NOT gates to produce an output only when input A is present alone. The specific NIMPLY gate design shown in Fig. $5 \mathrm{~g}$ uses the $\mathrm{ZnSO}_{4}$-induced RNA NOT gate alongside a DNA AND gate that requires both unregulated InvadeR and tetracycline-induced InvadeR for activation. Both the $\mathrm{ZnSO}_{4}$ NIMPLY tetracycline gate as well as the tetracycline NIMPLY $\mathrm{ZnSO}_{4}$ gate performed the expected logic gate computations (Fig. 5h and Extended Data Figs. 5k,l and 7k).

Together, these results show that basic logic gate components can be layered to perform more complex molecular computation using small molecules as inputs to the system.

Using a TMSD circuit to quantify biosensor outputs. To demonstrate a practical application of TMSD information processing, we next focused on quantifying biosensor outputs. In typical cell-free biosensing systems, outputs are generated when the input compound concentration is above a detection threshold, creating a 'presence/absence' results interpretation tuned to this threshold ${ }^{51}$. This detection threshold is determined by the aTF-ligand and aTFDNA binding constants, which can be difficult to tune. To address this limitation, we designed a system like an ADC circuit ${ }^{52}$ to create a series of binary outputs that encode the analog input concentration of the target compound (Supplementary Fig. 4).

To construct a genetic ADC circuit, we first created a comparator circuit-a building block of ADCs that produces a 'True' binary output when the input is above a predefined threshold. Thresholding is possible in TMSD because the reaction kinetics can be precisely increased by lengthening DNA gate toehold regions ${ }^{17}$ (Fig. 6a). Using this feature, we built an unlabeled DNA threshold gate that shares the same sequence with the DNA signal gate but with a longer eight-nucleotide toehold (Fig. 6a). Because the signal should be activated only after the threshold gate is completely consumed, we reasoned that by tuning the amount of the threshold gate, we can precisely control the time at which InvadeR activates fluorescence from the signal gate. Modeling this kinetic behavior showed that this is indeed the predicted behavior of the setup, and we experimentally observed quantitative agreement with modeling predictions (Fig. 6b). In this way, a thresholded TMSD reaction acts as a 'kinetic' comparator circuit-for a given input, the time at which signal generation occurs is proportional to the amount of the threshold gate.

Next, we created a series of biosensing TMSD comparator circuits to act as an ADC for ligand concentration by preparing a series of reactions in which each tube contains a different amount of the threshold gate. By adding the same ligand concentration to each tube, a user can observe which tubes in the series are activated at a specific time point and obtain semiquantitative information 


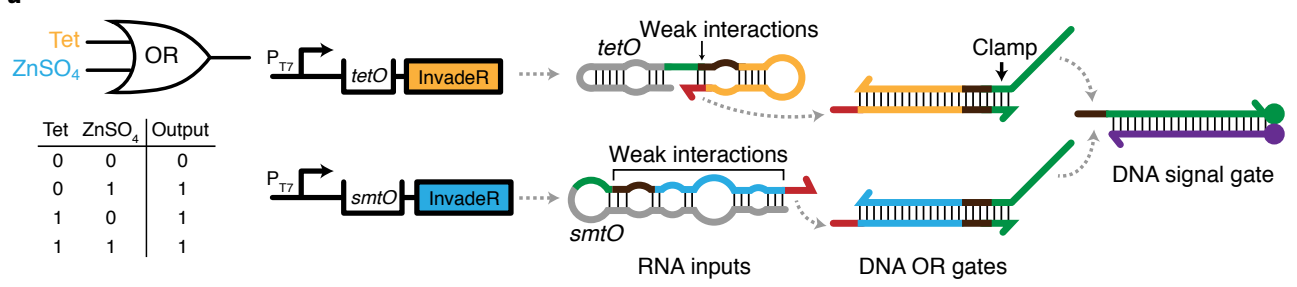

c
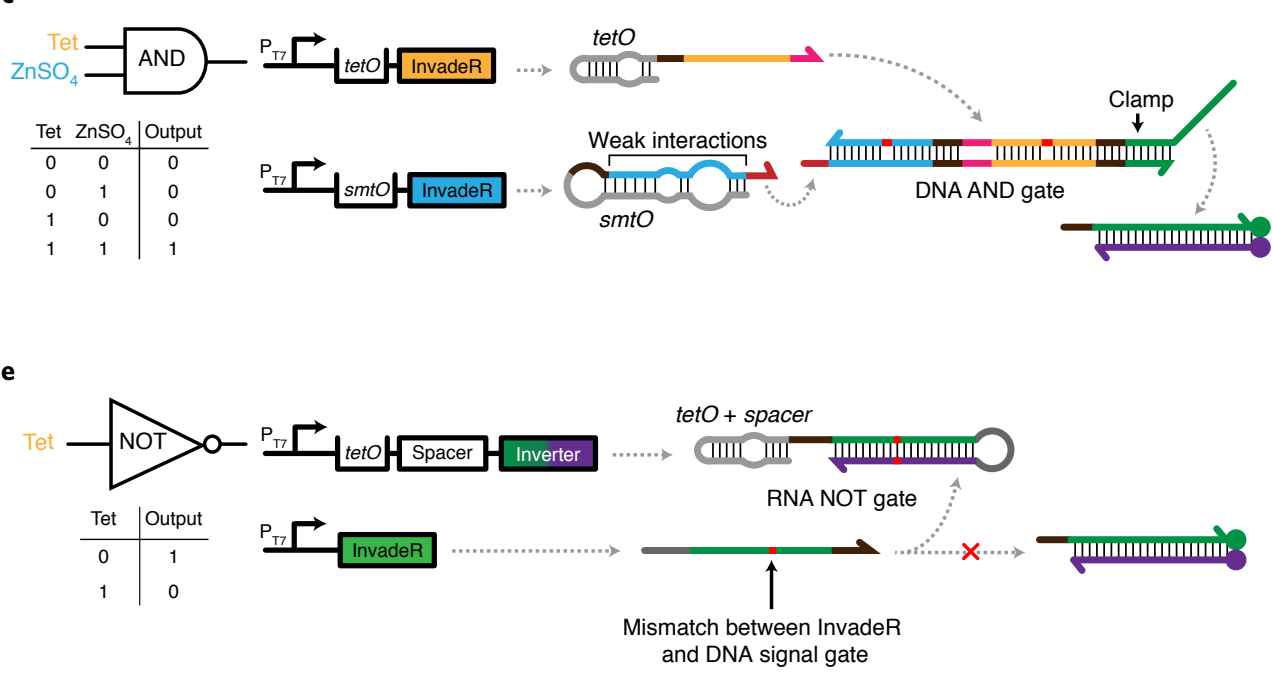
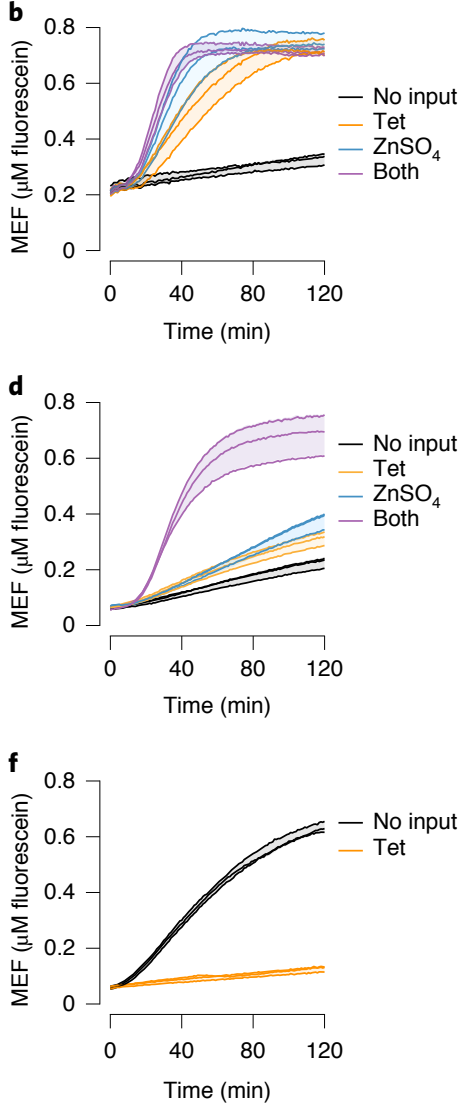

Fig. 4 | Additional gates can be layered to perform logic computation. a, A two-input OR gate includes two additional DNA OR gates. When transcription is activated by either ligand, InvadeR strands can react with their respective DNA OR gates to produce an output strand with a domain (green) that can invade the DNA signal gate. $\mathbf{b}$, When the two-input OR gate is activated by either tetracycline, $\mathrm{ZnSO}_{4}$ or both, fluorescence activation is observed. $\mathbf{c}$, $\mathrm{A}$ two-input AND gate contains a DNA AND gate that requires both InvadeR strands to dissociate the output strand to reveal the toehold complementary to the signal gate. Design features such as thermodynamic drivers (highlighted in red) and a clamp domain are implemented to facilitate efficient TMSD with minimal leak (Extended Data Fig. 6). d, The two-input AND gate activates fluorescence only when both tetracycline and $\mathrm{ZnSO}_{4}$ are present. e, A NOT gate includes two DNA templates: an unregulated InvadeR template and an aTF-regulated inverter template. Upon transcription, the aTF-regulated inverter forms a hairpin structure that resembles the DNA signal gate to create an RNA NOT gate. Unregulated InvadeR preferably reacts with the RNA NOT gate because of a greater number of base pair interactions and a base pair mismatch with the DNA signal gate (red). A spacer sequence is included to prevent the tetO sequence from interfering with the RNA NOT gate. $\mathbf{f}$, When implemented with a tetracycline sensor, the NOT gate generates signal in the absence of tetracycline. All data shown are $n=3$ independent biological replicates each plotted as a line with raw fluorescence value standardized to MEF ( $\mu$ M fluorescein). Shading indicates the average of the replicates \pm s.d. Domains with the same color share the same sequence except for the AND gate where the domain highlighted in orange is modified from the orange domain in the OR gate to improve its TMSD efficiency. All nucleic acid gates are drawn according to the secondary structures predicted using NUPACK at $37^{\circ} \mathrm{C}^{30}$. The sequence of each domain and the concentrations of components in each gate can be found in Supplementary Data 4 and 5, respectively.

about ligand concentration (Fig. 6c). We tested the feasibility of the approach using the same set of ODEs used in Fig. $6 \mathrm{~b}$ with the addition of aTF-DNA and aTF-ligand binding kinetics, focusing on zinc sensing because of its relevance in municipal water supplies $^{53}$. Using modeling simulations, we determined the threshold gate concentrations needed to activate one, two, three or four tubes after 100 min corresponding to zinc concentrations of 2, 3.5, 5 and $10 \mu \mathrm{M}$, respectively (Fig. 6d). We then built the corresponding genetic ADC circuit with the SmtB-regulated TMSD reactions and saw the expected pattern of signals after $100 \mathrm{~min}$, where the number of activated tubes in the series increased with higher input $\mathrm{ZnSO}_{4}$ concentrations (Fig. 6e and Extended Data Fig. 8a-d). This implementation allows a user to determine the input zinc concentration range by directly reading out the sequence of activated tubes.

We believe that this demonstration represents the potential for TMSD circuits to act as an information processing layer of cell-free biosensors that increase ease of interpretation and the information content of the output signals.

\section{Discussion}

In this study, we show that TMSD circuits can be interfaced with IVT to act as an information processing layer for cell-free biosensors. The speed of TMSD outputs led to a substantial enhancement of signal generation speed, with less than 10 min time-to-detection (Fig. 3f). More importantly, we found that the simple and defined nature of ROSALIND, combined with the computational power of TMSD enabled us to layer multiple RNA-DNA gates to build 12 different circuits that implement seven different logic functions in a modular fashion (Figs. 4 and 5). Harnessing this high programmability of the platform, we also designed and validated a circuit that can estimate the concentration range of an unknown target compound within a sample (Fig. 6). Finally, this platform is amenable to lyophilization (Extended Data Fig. 9) and can function with unprocessed real-world sample matrices (Extended Data Fig. 10).

The system presented a number of design challenges including the incompatibility of $3^{\prime}$ toeholds in DNA gates with T7 RNAP-driven IVT reactions (Extended Data Fig. 1) ${ }^{45}$, interfering 

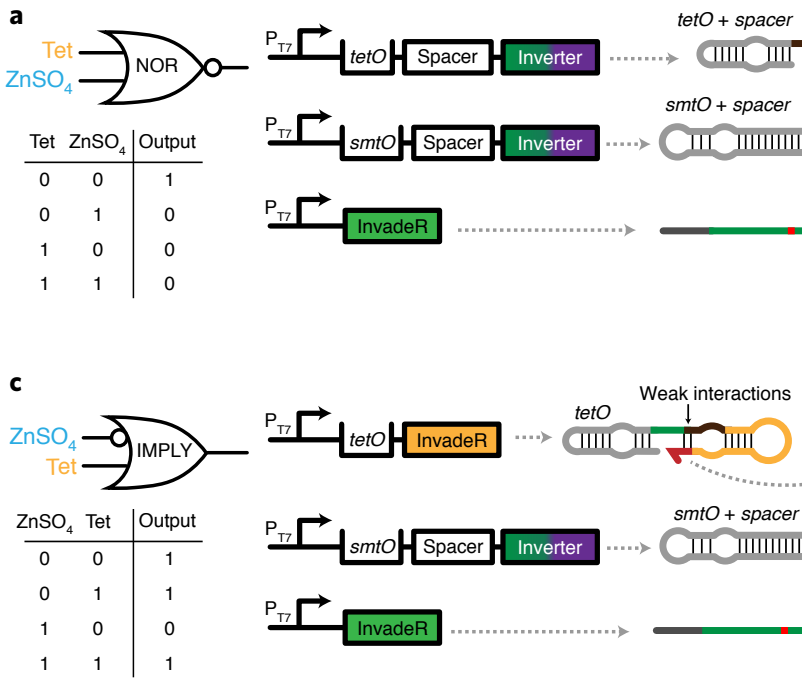

tetO + spacer

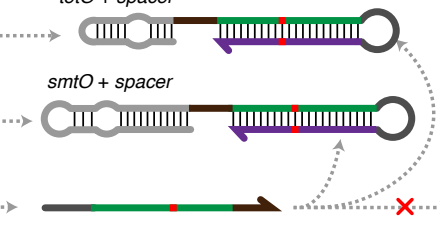

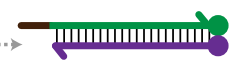

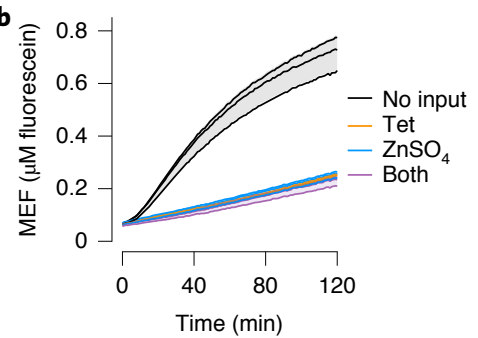

d

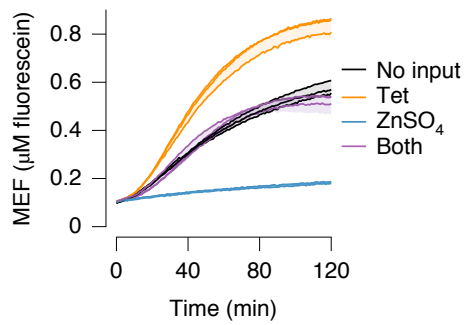

f

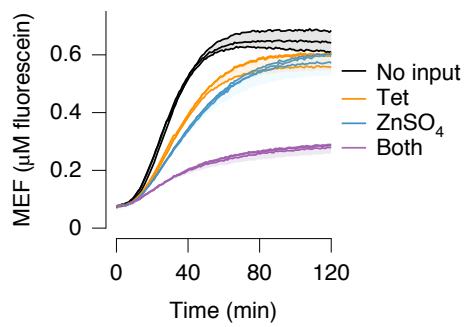

h

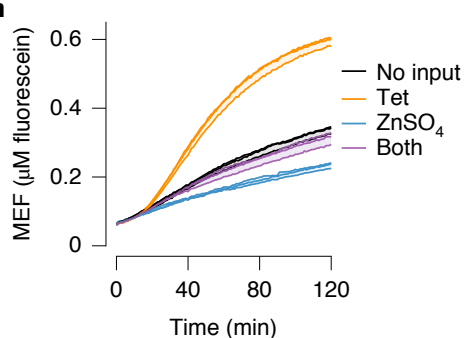

Fig. $\mathbf{5}$ | Logic component layering allows more complex computation. a, A two-input NOR gate is built by layering two RNA NOT gates. The NOT gates are regulated by either TetR or SmtB that sequester the unregulated InvadeR molecules from the DNA signal gate. $\mathbf{b}$, Fluorescence activation is observed only in the absence of both inputs. c, A two-input IMPLY gate combines a DNA OR gate with an RNA NOT gate. In this specific example (ZnSO IMPLY $_{4}$ tetracycline), the OR gate is regulated by TetR, and the NOT gate is regulated by $\mathrm{SmtB}$, preventing signal generation in the presence of $\mathrm{ZnSO}_{4}$ only. d, Fluorescence activation is observed unless only $\mathrm{ZnSO}_{4}$ is added. Faster signal generation is observed from the tetracycline only input condition due to no mismatch between the OR gate output strand and the signal gate. e, A two-input NAND gate layers two unregulated DNA OR gates with two regulated RNA NOT gates. In this configuration, the presence of both inputs is required to hinder signal generation. Thermodynamic drivers (highlighted in red) are incorporated in the NOT gates to favor the interactions with their respective InvadeR strands. $\mathbf{f}$, The expected NAND gate computation is observed. $\mathbf{g}$, A two-input NIMPLY gate is built by combining the DNA AND gate and the RNA NOT gate. In this specific example, tetracycline-induced InvadeR and unregulated InvadeR are required for the AND gate activation. A SmtB-regulated NOT gate sequesters the unregulated InvadeR. $\mathbf{h}$, Fluorescence activation is observed in the presence of tetracycline only. All data shown are $n=3$ independent biological replicates each plotted as a line with raw fluorescence value standardized to MEF ( $\mu \mathrm{M}$ fluorescein). Shading indicates the average of the replicates \pm s.d. Domains with the same color share the same sequence except for the AND gate where the domain highlighted in orange is modified from the orange domain in the OR gate. All nucleic acid gates are drawn according to the secondary structures predicted using NUPACK at $37^{\circ} \mathrm{C}^{30}$. The sequence of each domain and the concentrations of components in each gate can be found in Supplementary Data 4 and 5, respectively.

RNA secondary structures that can slow TMSD (Fig. 2 and Extended Data Fig. 4) and varying T7 transcription efficiency of different DNA templates ${ }^{36}$ (Extended Data Fig. 2). These challenges provided opportunities to develop RNA-based TMSD design principles that we believe are generalizable to other nucleic acid engineering systems (Supplementary Fig. 5).
One of the major limitations of the platform is its cost. Chemically modified oligos with purification can cost $~ 100$ USD or more, although a single batch can be used to make hundreds of reactions. Furthermore, DNA gates often need to be gel-purified after hybridization to eliminate any unbound ssDNA strands, which can be time-intensive and laborious. We note, however, that the cost 


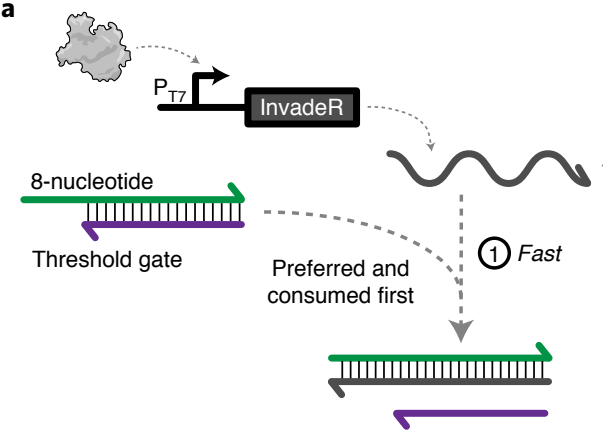

b

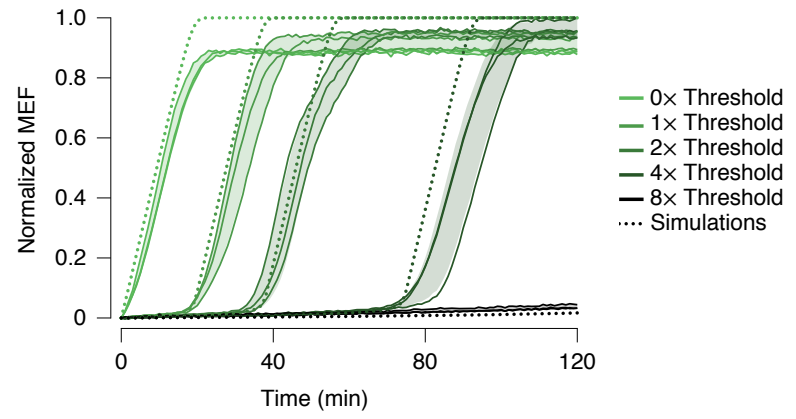

c Apply sample to a strip of tubes with varying threshold

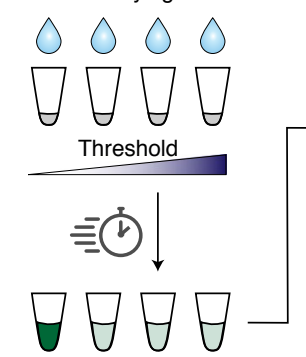

Number of ON tubes $=1$

\section{Compare with the standard to estimate [ligand]}

Threshold

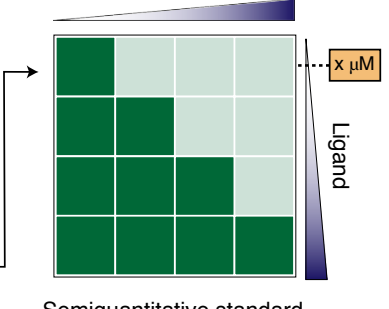

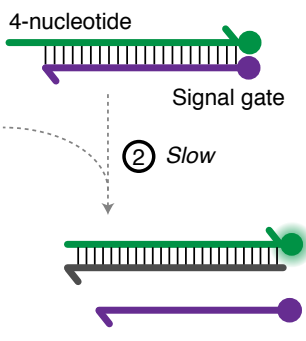

d

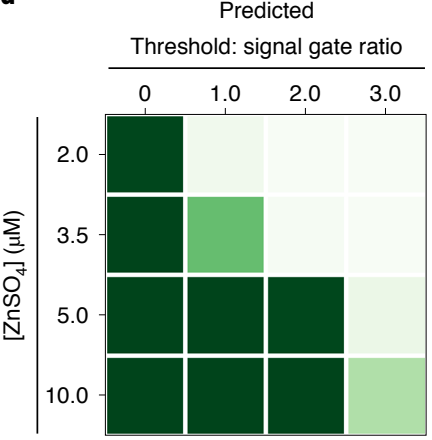

e

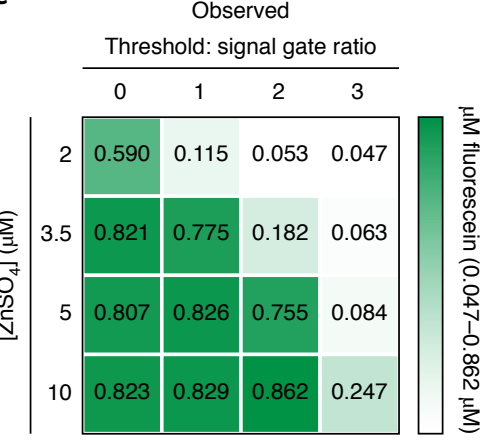

Fig. 6 | Quantifying ligand concentration with a genetic ADC circuit. a, Increasing the length of the DNA gate toehold region can be used to speed the strand invasion process. An unlabeled DNA gate with a longer toehold (eight nucleotides) can then preferentially react with InvadeR, acting as a programmable threshold. InvadeR can only strand-displace the signal gate (four-nucleotide toehold) after the threshold gate is exhausted. $\mathbf{b}$, Titrating the eight-nucleotide toehold threshold gate in different ratios above a fixed signal gate concentration $(0-8 x)$ results in a time delay in fluorescence activation that can be quantitatively modeled with ODE simulations (dotted lines). All data shown for $n=3$ independent biological replicates each plotted as a line. Raw fluorescence values were first standardized to MEF ( $\mu \mathrm{M}$ fluorescein) and normalized to the maximum MEF among all conditions to accommodate their comparison with the simulations (See Methods for the normalization method used). Shading indicates the average of the replicates \pm s.d. $\mathbf{c}, A$ molecular ADC circuit is made by constructing a strip of tests of the same sensor, with each test containing a different concentration of the DNA threshold gate. A higher threshold gate concentration requires a higher ligand concentration to activate fluorescence. When the same sample is applied to each tube, a user can obtain semiquantitative information about the concentration of ligand present in the sample (analog input) by identifying the series of tubes that activate (binary digital output). Here, we define a tube with its MEF value $>0.5$ as 'ON' because the visible threshold is around the indicated value. $\mathbf{d}, \mathbf{e}$, Characterization of a molecular ADC circuit for zinc using ODE simulations (d) and endpoint experimental data at 100 min (e) generated using the SmtB-regulated zinc sensor. The values on the heatmap represent the average MEF ( $\mu$ M fluorescein) of $n=3$ independent biological replicates (see Extended Data Fig. 8 for all data).

of chemical dyes for fluorescence-activating RNA aptamer reporting systems is not negligible, and the advantages provided by the TMSD system such as the improved response speed and computational power outcompete its limitations.

The key feature of this study was demonstrating the potential of TMSD circuits to expand the function of cell-free biosensors by acting as information processing layers. While an approach was recently developed to interface aTF-based biosensing with TMSD through endonuclease-mediated TMSD cascades $^{54}$, no programmable molecular computation beyond simple contaminant detection was presented. As a demonstration, we modeled, designed and validated several layered RNA-based TMSD circuits capable of performing complex logic gate computation with small molecule inputs by adapting several elements of DNA-based TMSD logic gates (Figs. 4 and 5, and Extended Data Figs. 5-7). Although not shown, more complex logic circuits such as XOR and XNOR could be designed by layering additional gates.

To further highlight the platform's capability for information processing, we developed a genetic ADC circuit that can be used to estimate an input ligand concentration at a semiquantitative level (Fig. 6). In particular, this circuit uses thresholding computation to convert an analog signal of an input molecule concentration into a digital output of the number of activated tubes. This development was enabled by the ability to precisely tune reaction rates of
TMSD on the basis of the toehold length. We note, however, this genetic ADC circuit is different from an electrical ADC circuit in that its result depends on time of activation and not activation level, because the circuit relies on thresholding reaction kinetics rather than strictly input concentrations. As a result, this strategy is best suited to distinguishing between ligand concentrations that cause differences in output kinetics (Extended Data Fig. 8e-g).

We believe that this platform opens the door to other types of molecular computation in cell-free systems. For example, an amplification circuit such as a catalytic hairpin assembly ${ }^{55}$ could be applied to ROSALIND with TMSD for amplifying signals and making a sensor ultrasensitive. Beyond thresholding, other operations demonstrated in DNA seesaw gate architectures could be ported to this platform for various computations ${ }^{46}$. Specifically, logic gates can be extended to develop a general strategy to fix aTF ligand promiscuity ${ }^{7}$. In addition, because virtually any aTF that functions in an in vitro context can be used ${ }^{7}$, multiple DNA gates with different reporters could be added for multiplexing. The fundamental role that ADC circuits play in interfacing analog and digital electronic circuitry also holds promise for adopting additional electronic circuit designs to biochemical reactions.

Together, these results show that establishing an interface between small molecule biosensing and TMSD circuits is a promising first step towards creating a general molecular computation 
platform to enhance and expand the function of cell-free biosensing technologies.

\section{Online content}

Any methods, additional references, Nature Research reporting summaries, source data, extended data, supplementary information, acknowledgements, peer review information; details of author contributions and competing interests; and statements of data and code availability are available at https://doi.org/10.1038/s41589-021-00962-9.

Received: 29 January 2021; Accepted: 16 December 2021; Published online: 17 February 2022

\section{References}

1. Thavarajah, W. et al. Point-of-use detection of environmental fluoride via a cell-free riboswitch-based biosensor. ACS Synth. Biol. 9, 10-18 (2020).

2. Silverman, A. D. et al. Design and optimization of a cell-free atrazine biosensor. ACS Synth. Biol. 9, 671-677 (2020).

3. Pardee, K. Perspective: Solidifying the impact of cell-free synthetic biology through lyophilization. Biochem. Eng. J. 138, 91-97 (2018).

4. McNerney, M. P. et al. Point-of-care biomarker quantification enabled by sample-specific calibration. Sci. Adv. 5, eaax4473 (2019).

5. Wen, K. Y. et al. A cell-free biosensor for detecting quorum sensing molecules in P. aeruginosa-infected respiratory samples. ACS Synth. Biol. 6, 2293-2301 (2017).

6. Grawe, A. et al. A paper-based, cell-free biosensor system for the detection of heavy metals and date rape drugs. PLoS ONE 14, e0210940 (2019).

7. Jung, J. K. et al. Cell-free biosensors for rapid detection of water contaminants. Nat. Biotechnol. 38, 1451-1459 (2020).

8. Alon, U. An Introduction to Systems Biology: Design Principles of Biological Circuits 2nd edn (CRC Press, 2019).

9. Nielsen, A. A. et al. Genetic circuit design automation. Science 352, aac7341 (2016).

10. Wan, X. et al. Cascaded amplifying circuits enable ultrasensitive cellular sensors for toxic metals. Nat. Chem. Biol. 15, 540-548 (2019).

11. Zhang, D. Y. \& Seelig, G. Dynamic DNA nanotechnology using strand-displacement reactions. Nat. Chem. 3, 103-113 (2011).

12. Qian, L. \& Winfree, E. A simple DNA gate motif for synthesizing large-scale circuits. J. R. Soc. Interface 8, 1281-1297 (2011).

13. Zhang, D. Y. et al. Engineering entropy-driven reactions and networks catalyzed by DNA. Science 318, 1121-1125 (2007).

14. Li, W. et al. Three-input majority logic gate and multiple input logic circuit based on DNA strand displacement. Nano Lett. 13, 2980-2988 (2013).

15. Seelig, G. et al. Enzyme-free nucleic acid logic circuits. Science 314, 1585-1588 (2006).

16. Hjelmfelt, A., Weinberger, E. D. \& Ross, J. Chemical implementation of neural networks and Turing machines. Proc. Natl Acad. Sci. USA 88, 10983-10987 (1991).

17. Zhang, D. Y. \& Winfree, E. Control of DNA strand displacement kinetics using toehold exchange. J. Am. Chem. Soc. 131, 17303-17314 (2009).

18. Kim, J. \& Winfree, E. Synthetic in vitro transcriptional oscillators. Mol. Syst. Biol. 7, 465 (2011)

19. Jiang, Y. S. et al. Real-time detection of isothermal amplification reactions with thermostable catalytic hairpin assembly. J. Am. Chem. Soc. 135, 7430-7433 (2013).

20. Yurke, B. et al. A DNA-fuelled molecular machine made of DNA. Nature 406 605-608 (2000).

21. Dirks, R. M. \& Pierce, N. A. Triggered amplification by hybridization chain reaction. Proc. Natl Acad. Sci. USA 101, 15275-15278 (2004).

22. Yin, P. et al. Programming biomolecular self-assembly pathways. Nature 451, 318-322 (2008).

23. Green, L. N. et al. Autonomous dynamic control of DNA nanostructure self-assembly. Nat. Chem. 11, 510-520 (2019).

24. $\mathrm{Hu}, \mathrm{X}$. et al. Single-molecule catalytic hairpin assembly for rapid and direct quantification of circulating miRNA biomarkers. Anal. Chim. Acta 1042, 109-115 (2018).

25. Jiang, Y. S. et al. Robust strand exchange reactions for the sequence-specific, real-time detection of nucleic acid amplicons. Anal. Chem. 87, 3314-3320 (2015)

26. Jiang, Y. S. et al. Portable platform for rapid in-field identification of human fecal pollution in water. Water Res. 131, 186-195 (2018).

27. Schaffter, S. W. et al. T7 RNA polymerase non-specifically transcribes and induces disassembly of DNA nanostructures. Nucleic Acids Res. 46 , 5332-5343 (2018).

28. Liu, H. et al. Kinetics of RNA and RNA:DNA hybrid strand displacement. ACS Synth. Biol. 10, 3066-3073 (2021).
29. Bhadra, S. \& Ellington, A. D. Design and application of cotranscriptional non-enzymatic RNA circuits and signal transducers. Nucleic Acids Res. 42, e58 (2014).

30. Zadeh, J. N. et al. NUPACK: Analysis and design of nucleic acid systems. J. Comput. Chem. 32, 170-173 (2011).

31. Sharmeen, L. \& Taylor, J. Enzymatic synthesis of RNA oligonucleotides. Nucleic Acids Res. 15, 6705-6711 (1987).

32. Krupp, G. Unusual promoter-independent transcription reactions with bacteriophage RNA polymerases. Nucleic Acids Res. 17, 3023-3036 (1989).

33. Marras, S. et al. Real-time measurement of in vitro transcription. Nucleic Acids Res. 32, e72 (2004).

34. Srinivas, N. et al. On the biophysics and kinetics of toehold-mediated DNA strand displacement. Nucleic Acids Res. 41, 10641-10658 (2013).

35. Sulc, P. et al. Modelling toehold-mediated RNA strand displacement. Biophys. J. 108, 1238-1247 (2015).

36. Conrad, T. et al. Maximizing transcription of nucleic acids with efficient T7 promoters. Commun. Biol 3, 439 (2020).

37. Cuthbertson, L. \& Nodwell, J. R. The TetR family of regulators. Microbiol. Mol. Biol. Rev. 77, 440-475 (2013).

38. Han, K. Y. et al. Understanding the photophysics of the spinach-DFHBI RNA aptamer-fluorogen complex to improve live-cell RNA imaging. J. Am. Chem. Soc. 135, 19033-19038 (2013).

39. Mitra, J. \& Ha, T. Nanomechanics and co-transcriptional folding of Spinach and Mango. Nat. Commun. 10, 4318 (2019).

40. Rogers, J. K. et al. Synthetic biosensors for precise gene control and real-time monitoring of metabolites. Nucleic Acids Res. 43, 7648-7660 (2015).

41. VanZile, M. L., Chen, X. \& Giedroc, D. P. Allosteric negative regulation of smt $\mathrm{O} / \mathrm{P}$ binding of the zinc sensor, $\mathrm{SmtB}$, by metal ions: a coupled equilibrium analysis. Biochemistry 41, 9776-9786 (2002).

42. Deochand, D. K. \& Grove, A. MarR family transcription factors: dynamic variations on a common scaffold. Crit. Rev. Biochem. Mol. Biol. 52, 595-613 (2017).

43. Busenlehner, L. S., Pennella, M. A. \& Giedroc, D. P. The SmtB/ArsR family of metalloregulatory transcriptional repressors: Structural insights into prokaryotic metal resistance. FEMS Microbiol. Rev. 27, 131-143 (2003).

44. Krishnan, Y. \& Simmel, F. C. Nucleic acid based molecular devices. Angew. Chem. Int. Ed. Engl. 50, 3124-3156 (2011).

45. Schaffter, S. W. \& Schulman, R. Building in vitro transcriptional regulatory networks by successively integrating multiple functional circuit modules. Nat Chem. 11, 829-838 (2019).

46. Qian, L. \& Winfree, E. Scaling up digital circuit computation with DNA strand displacement cascades. Science 332, 1196-1201 (2011).

47. Schaffter, S. W. \& Strychalski, E. A. Co-transcriptional RNA strand displacement circuits. Preprint at bioRxiv https://doi. org/10.1101/2021.07.20.450530 (2021)

48. Haley, N. E. C. et al. Design of hidden thermodynamic driving for non-equilibrium systems via mismatch elimination during DNA strand displacement. Nat. Commun. 11, 2562 (2020).

49. Wang, B. et al. Effective design principles for leakless strand displacement systems. Proc. Natl Acad. Sci. USA 115, E12182-E12191 (2018).

50. Bae, W., Stan, G. V. \& Ouldridge, T. E. In situ generation of RNA complexes for synthetic molecular strand-displacement circuits in autonomous systems. Nano Lett. 21, 265-271 (2021).

51. Mannan, A. A. et al. Fundamental design principles for transcription-factorbased metabolite biosensors. ACS Synth. Biol. 6, 1851-1859 (2017).

52. Ida, N. Sensors, Actuators, and Their Interfaces: A Multidisciplinary Introduction (SciTech Publishing, 2014).

53. World Health Organization. Guidelines for Drinking-water Quality 4th edn (World Health Organization, 2011).

54. Rodriguez-Serrano, A. F. \& Hsing, I. M. Allosteric regulation of DNA circuits enables minimal and rapid biosensors of small molecules. ACS Synth. Biol. 10, 371-378 (2021).

55. Liu, J. et al. Applications of catalytic hairpin assembly reaction in biosensing. Small 15, e1902989 (2019).

Publisher's note Springer Nature remains neutral with regard to jurisdictional claims in published maps and institutional affiliations.

Open Access This article is licensed under a Creative Commons

Attribution 4.0 International License, which permits use, sharing, adaptation, distribution and reproduction in any medium or format, as long as you give appropriate credit to the original author(s) and the source, provide a link to the Creative Commons license, and indicate if changes were made. The images or other third party material in this article are included in the article's Creative Commons license, unless indicated otherwise in a credit line to the material. If material is not included in the article's Creative Commons license and your intended use is not permitted by statutory regulation or exceeds the permitted use, you will need to obtain permission directly from the copyright holder. To view a copy of this license, visit http://creativecommons. org/licenses/by/4.0/.

(c) The Author(s) 2022 


\section{Methods}

Strains and growth medium. Escherichia coli strain K12 (NEB Turbo Competent E. coli, New England Biolabs, catalog no. C2984) was used for routine cloning. E. coli strain Rosetta 2(DE3)pLysS (Novagen, catalog no. 71401) was used for recombinant protein expression. Luria broth supplemented with the appropriate antibiotic(s) $\left(100 \mu \mathrm{g} \mathrm{ml}^{-1}\right.$ carbenicillin, $100 \mu \mathrm{g} \mathrm{ml}^{-1}$ kanamycin and/or $34 \mu \mathrm{g} \mathrm{ml}^{-1}$ chloramphenicol) was used as the growth media.

DNA gate preparation. DNA signal gates used in this study were synthesized by Integrated DNA technologies as modified oligos. They were generated by denaturing a 6-carboxyfluorescein (6-FAM) modified oligonucleotide and the complementary Iowa Black FQ quencher modified oligonucleotide (Supplementary Data 1$)$ at $95^{\circ} \mathrm{C}$ separately for $3 \mathrm{~min}$ and slow cooling $\left(-0.1^{\circ} \mathrm{C} \mathrm{s}^{-1}\right)$ to room temperature in annealing buffer $(100 \mathrm{mM}$ potassium acetate and $30 \mathrm{mM}$ HEPES, $\mathrm{pH}$ 8.0). Annealed oligonucleotides where then purified by resolving them on $20 \%$ native PAGE-Tris-Borate-EDTA (TBE) gels, isolating the band of expected size and eluting at $4{ }^{\circ} \mathrm{C}$ overnight in annealing buffer. The eluted DNA gate was then ethanol-precipitated, resuspended in MilliQ ultrapure $\mathrm{H}_{2} \mathrm{O}$ and concentration quantified using the Thermo Scientific NanoDrop One Microvolume UV-Vis Spectrophotometer. The DNA gates used in Figs. 4-6 and Extended Data Fig. 6 and 7 were prepared using the same method but by annealing two complementary oligonucleotides without any modifications.

Plasmids and genetic parts assembly. DNA oligonucleotides for cloning and sequencing were synthesized by Integrated DNA Technologies. Genes encoding aTFs were synthesized either as gBlocks (Integrated DNA Technologies) or gene fragments (Twist Bioscience). Protein expression plasmids were cloned using Gibson Assembly (NEB Gibson Assembly Master Mix, New England Biolabs, catalog no. E2611) into a pET-28c plasmid backbone and were designed to overexpress recombinant proteins as $\mathrm{C}$ terminus His-tagged fusions. A construct for expressing $\mathrm{SmtB}$ additionally incorporated a recognition sequence for cleavage and removal of the His-tag using TEV protease. Gibson assembled constructs were transformed into NEB Turbo cells, and isolated colonies were purified for plasmid DNA (QIAprep Spin Miniprep Kit, Qiagen, catalog no. 27106). Plasmid sequences were verified with Sanger DNA sequencing (Quintara Biosciences) using the primers listed in Supplementary Data 1.

All transcription templates were generated using one of the two methods: (1) PCR amplification (Phusion High-Fidelity PCR Kit, New England Biolabs, catalog no. E0553) of an oligo that includes a T7 promoter, an optional aTF operator site, the InvadeR coding sequence and an optional T7 terminator using the primer sets; or (2) annealing of two complementary oligonucleotides that include a T7 promoter, an optional aTF operator site, the InvadeR or RNA NOT gate coding sequences. All oligos and primer sets used in this study are listed in listed in Supplementary Data 1. Here, we define the T7 promoter as a minimal 17-bp sequence (TAATACGACTCACTATA) excluding the first $\mathrm{G}$ that is transcribed. The PCR-amplified templates were purified (QIAquick PCR purification kit, Qiagen, catalog no. 28106) and verified for the presence of a single DNA band of expected size on a $2 \%$ Tris-Acetate-EDTA-agarose gel. The templates generated by annealing two complementary oligos were prepared and purified using the same method described in 'DNA gate preparation'. Concentrations of all DNA templates were determined using the Qubit dsDNA BR Assay Kit (Invitrogen, catalog no. Q32853).

All plasmids and DNA templates were stored at $4{ }^{\circ} \mathrm{C}$ until use. A spreadsheet listing the sequences and the Addgene accession numbers of all plasmids and oligos generated in this study are listed in Supplementary Data 1.

RNA expression and purification. InvadeR variants used for the purified oligo binding assays were first expressed by an overnight IVT at $37^{\circ} \mathrm{C}$ from a transcription template encoding a cis-cleaving hepatitis $\mathrm{D}$ ribozyme on the 3 '-end of the InvadeR sequence with the following components: IVT buffer (40 mM Tris- $\mathrm{HCl} \mathrm{pH} 8,8 \mathrm{mM} \mathrm{MgCl}_{2}, 10 \mathrm{mM}$ dithiothreitol, $20 \mathrm{mM} \mathrm{NaCl}$ and $2 \mathrm{mM}$ spermidine), $11.4 \mathrm{mM}$ NTPs pH 7.5, 0.3 units (U) of thermostable inorganic pyrophosphatase (New England Biolabs, catalog no. M0296S), $100 \mathrm{nM}$ transcription template, $50 \mathrm{ng}$ of T7 RNAP and MilliQ ultrapure $\mathrm{H}_{2} \mathrm{O}$ to a total volume of $500 \mu \mathrm{l}$. The overnight IVT reactions were then ethanol-precipitated and purified by resolving them on a $20 \%$ urea-PAGE-TBE gel, isolating the band of expected size (26-29 nucleotides) and eluting at $4{ }^{\circ} \mathrm{C}$ overnight in MilliQ ultrapure $\mathrm{H}_{2} \mathrm{O}$. The eluted InvadeR variants were ethanol-precipitated, resuspended in MilliQ ultrapure $\mathrm{H}_{2} \mathrm{O}$, quantified using the Qubit RNA BR Assay Kit (Invitrogen, catalog no. Q10211) and stored at $-20^{\circ} \mathrm{C}$ until use. The hepatitis D ribozyme sequence used can be found in Supplementary Data 1.

aTF expression and purification. aTFs were expressed and purified as previously described $^{7}$. Briefly, sequence-verified pET-28c plasmids were transformed into the Rosetta 2(DE3)pLysS E. coli strain. Cell cultures (1-2L) were grown in Luria broth at $37^{\circ} \mathrm{C}$, induced with $0.5 \mathrm{mM}$ of isopropyl- $\beta$-D-thiogalactoside at an optical density $(600 \mathrm{~nm})$ of $\sim 0.5$ and grown for a further $4 \mathrm{~h}$ at $37^{\circ} \mathrm{C}$. Cultures were then pelleted by centrifugation and were either stored at $-80^{\circ} \mathrm{C}$ or resuspended in lysis buffer (10 mM Tris- $\mathrm{HCl}$ pH 8, $500 \mathrm{mM} \mathrm{NaCl}, 1 \mathrm{mM}$ Tris(2-carboxyethyl) phosphine (TCEP) and protease inhibitor (Complete EDTA-free Protease Inhibitor
Cocktail, Roche)) for purification. Resuspended cells were then lysed on ice through ultrasonication, and insoluble materials were removed by centrifugation. Clarified supernatant containing TetR was then purified using His-tag affinity chromatography with a Ni-NTA column (HisTrap FF $5 \mathrm{ml}$ column, GE Healthcare Life Sciences) followed by size-exclusion chromatography (Superdex HiLoad 26/600 200 pg column, GE Healthcare Life Sciences) using an AKTAxpress fast protein liquid chromatography system. Clarified supernatants containing TtgR and SmtB were purified using His-tag affinity chromatography with a gravity column charged with Ni-NTA Agarose (Qiagen, catalog no. 30210). The eluted fractions from the fast protein liquid chromatography (for TetR) or from the gravity column (for TtgR and SmtB) were concentrated and buffer exchanged $(25 \mathrm{mM}$ Tris- $\mathrm{HCl}$, $100 \mathrm{mM} \mathrm{NaCl}, 1 \mathrm{mM}$ TCEP, 50\% glycerol v/v) using centrifugal filtration (Amicon Ultra-0.5, Millipore Sigma). Protein concentrations were determined using the Qubit Protein Assay Kit (Invitrogen, catalog no. Q33212). The purity and size of the proteins were validated on an SDS-PAGE gel (Mini-PROTEAN TGX and Mini-TETRA cell, Bio-Rad). Purified proteins were stored at $-20^{\circ} \mathrm{C}$.

IVT reactions. Homemade IVT reactions were set up by adding the following components listed at their final concentration: IVT buffer $(40 \mathrm{mM}$ Tris- $\mathrm{HCl} \mathrm{pH} 8$, $8 \mathrm{mM} \mathrm{MgCl}_{2}, 10 \mathrm{mM}$ dithiothreitol, $20 \mathrm{mM} \mathrm{NaCl}$ and $2 \mathrm{mM}$ spermidine), $11.4 \mathrm{mM}$ NTPs pH 7.5, $0.3 \mathrm{U}$ of thermostable inorganic pyrophosphatase (New England Biolabs, catalog no. M0296S), transcription template, DNA gate(s) and MilliQ ultrapure $\mathrm{H}_{2} \mathrm{O}$ to a total volume of $20 \mu \mathrm{l}$. Regulated IVT reactions additionally included a purified aTF at the indicated concentration and were equilibrated at $37^{\circ} \mathrm{C}$ for $\sim 10 \mathrm{~min}$. Immediately before plate reader measurements, $2 \mathrm{ng}$ of T7 RNAP and, optionally, a ligand at the indicated concentration were added to the reaction. Reactions were then characterized on a plate reader as described in 'Plate reader quantification and micromolar equivalent fluorescein standardization'.

RNA extraction from IVT reactions. For RNA products shown on the gel images of Extended Data Figs. 1c,f and 2c, IVT reactions were first set up as described above. Then, phenol-chloroform extraction followed by ethanol precipitation was performed to remove any proteins. The reactions were then rehydrated in $1 \times$ TURBO DNase buffer with $2 \mathrm{U}$ of TURBO DNase (Invitrogen, catalog no. QAM2238) to a total volume of $20 \mu \mathrm{l}$ and incubated at $37^{\circ} \mathrm{C}$ for $30 \mathrm{~min}$ to remove the DNA gates and the transcription templates. Phenol-chloroform extraction followed by ethanol precipitation was performed again to remove DNase and rehydrated in MilliQ ultrapure $\mathrm{H}_{2} \mathrm{O}$. The concentrations of the extracted RNA products were measured using the Qubit RNA HS assay kit (Invitrogen, catalog no. Q32852) and stored in $-20^{\circ} \mathrm{C}$ until further analysis such as PAGE. For PAGE analysis of these extracted RNA products, $20 \%$ urea-PAGE-TBE gels were used, and the gels were imaged using a ChemiDoc Touch Gel Imaging System (Bio-Rad Image Lab Touch software v.1.2.0.12).

Freeze-drying. Before lyophilization, PCR tube caps were punctured with a pin to create three holes. Lyophilization of ROSALIND reactions was then performed by assembling the components of IVT (above) with the addition of $50 \mathrm{mM}$ sucrose and $250 \mathrm{mM}$ D-mannitol. Assembled reaction tubes were immediately transferred into a prechilled aluminum block and placed in a $-80^{\circ} \mathrm{C}$ freezer for $10 \mathrm{~min}$ to allow slow-freezing. Following the slow-freezing, reaction tubes were wrapped in Kimwipes and aluminum foil, submerged in liquid nitrogen and then transferred to a FreeZone 2.5L Bench Top Freeze Dry System (Labconco) for overnight freeze-drying with a condenser temperature of $-85^{\circ} \mathrm{C}$ and $0.04 \mathrm{mbar}$ pressure. Unless rehydrated immediately, freeze-dried reactions were packaged as follows. The reactions were placed in a vacuum-sealable bag with a desiccant (Dri-Card Desiccants, Uline, catalog no. S-19582), purged with argon using an argon canister (ArT Wine Preserver, Amazon, catalog no. 8541977939) and immediately vacuum-sealed (KOIOS Vacuum Sealer Machine, Amazon, catalog no. TVS-2233). The vacuum-sealed bag then was placed in a light-protective bag (Mylar open-ended food bags, Uline, catalog no. S-11661), heat-sealed (Metronic 8-inch Impulse Bag Sealer, Amazon, catalog no. 8541949845) and stored in a cool, shaded area until use.

\section{Plate reader quantification and micromolar equivalent fluorescein} standardization. A National Institute of Standards and Technology traceable standard (Invitrogen, catalog no. F36915) was used to convert arbitrary fluorescence measurements to micromolar equivalent fluorescein (MEF). Serial dilutions from a $50 \mu \mathrm{M}$ stock were prepared in $100 \mathrm{mM}$ sodium borate buffer at $\mathrm{pH} 9.5$, including a $100 \mathrm{mM}$ sodium borate buffer blank (total of 12 samples). For each concentration, nine replicates of samples were created in batches of three, and fluorescence values were read at an excitation wavelength of $495 \mathrm{~nm}$ and emission wavelength of $520 \mathrm{~nm}$ for 6-FAM (fluorescein)-activated fluorescence, or at an excitation wavelength of $472 \mathrm{~nm}$ and emission wavelength of $507 \mathrm{~nm}$ for 3-way junction dimeric broccoli (3WJdB)-activated fluorescence on a plate reader (Synergy H1, BioTek Gen5 v.2.04). Fluorescence values for a fluorescein concentration in which a single replicate saturated the plate reader were excluded from the analysis. The remaining replicates (nine per sample) were then averaged at each fluorescein concentration, and the average fluorescence value of the blank was subtracted from all values. Linear regression was then performed for concentrations within the linear range of fluorescence $(0-3.125 \mu \mathrm{M}$ fluorescein) 
between the measured fluorescence values in arbitrary units and the concentration of fluorescein to identify the conversion factor. For each plate reader, excitation, emission and gain setting, we found a linear conversion factor that was used to correlate arbitrary fluorescence values to MEF (Supplementary Fig. 1 and Supplementary Data 3).

For characterization, $19 \mu \mathrm{l}$ of reactions were loaded onto a 384 -well optically clear, flat-bottom plate using a multichannel pipette, covered with a plate seal and measured on a plate reader (Synergy H1, BioTek Gen5 v.2.04). Kinetic analysis of 6-FAM (fluorescein)-activated fluorescence was performed by reading the plate at 1-min intervals with excitation and emission wavelengths of 495 and $520 \mathrm{~nm}$, respectively, for $2 \mathrm{~h}$ at $37^{\circ} \mathrm{C}$. Kinetic analysis of $3 \mathrm{WJdB}$-activated fluorescence was performed by reading the plate at 3-min intervals with excitation and emission wavelengths of 472 and $507 \mathrm{~nm}$, respectively, for $4 \mathrm{~h}$ at $37^{\circ} \mathrm{C}$. Arbitrary fluorescence values were then converted to MEF by dividing with the appropriate calibration conversion factor.

Except for the data in Fig. 6b, no background subtraction was performed when analyzing outputs from any reaction. An example of this standardization procedure is shown in Supplementary Fig. 1.

Fluorescence data normalization (Fig. 6b only). Data shown in Fig. 6b were generated as above and then normalized using the following method to compare experimental observations with ODE simulations. Raw fluorescence values were first standardized to MEF ( $\mu \mathrm{M}$ fluorescein) using the method described above. The maximum MEF value was then determined among all of the reactions run (5 conditions $\times 3$ replicates $=15$ reactions). Each MEF value at every time interval was then normalized using the following formula:

$$
f(x)=\frac{\mathrm{MEF}_{t=x}-\mathrm{MEF}_{t=0}}{\operatorname{Max} \mathrm{MEF}-\mathrm{MEF}_{t=0}}
$$

where $x$ is a given time point $(0 \leq x \leq 120)$

Background subtraction was performed to account for the non-zero fluorescence observed for the quenched DNA signal gate. Once all data were normalized according to the formula above, $n=3$ replicates per condition were averaged, and the corresponding standard deviation value per condition was calculated.

Gel image analysis. Uncropped, unprocessed gel images presented in Supplementary Fig. $2 \mathrm{~d}$ and Extended Data Figs. 1c,f and $2 \mathrm{c}$ are available either as source data or as Supplementary Data 2 and deposited in Mendeley Data (https:// doi.org/10.17632/hr3j3yztxb.1). The band intensity from a SYBR gold-stained urea-PAGE gel in Extended Data Fig. 2c was calculated with Fiji-ImageJ using the traditional lane-profile method as previously described ${ }^{56}$. Briefly, a region of interest in every lane was registered using a rectangle of the same dimensions. The uneven background was then accounted for by drawing a straight line at the bottom of each peak, and the peak area in each lane was calculated using the wand tool. The peak areas of the RNA standard were then plotted against the total amounts loaded to create the standard curve in Extended Data Fig. $2 \mathrm{~d}$ (linear range: $0.25-2 \mathrm{ng}$ ). Using the conversion factor from the standard curve, the concentrations of InvadeR variants were estimated from the peak area values obtained from the wand tool.

Tap and lake water sampling. For $\mathrm{ZnSO}_{4}$-spiked tap water from Evanston, IL, two bottles containing approximately $50 \mathrm{ml}$ of the water samples were collected from a drinking fountain. One of the bottles was then filtered at $0.22 \mu \mathrm{m}$ using a Steriflip-GP sterile vacuum filtration system (Millipore Sigma, catalog no. SCGP00525). Both the filtered and unfiltered water samples were spiked using 10 , 1 or $0.1 \mathrm{mM} \mathrm{ZnSO}_{4}$ solution that has been diluted from the $2 \mathrm{M} \mathrm{ZnSO}_{4}$ solution stock (Sigma, catalog no. 83265). Upon rehydration, fluorescence measurements of the reactions were performed using a plate reader (see 'Plate reader quantification and MEF standardization'). For $\mathrm{ZnSO}_{4}$-spiked Lake Michigan water from Evanston, IL, the same sampling method was applied.

ODE simulations. ODEs for each reactant species were derived using aTF-binding, IVT and TMSD reaction kinetics. ODEs were calculated using an ODE solver function, odeint from the Scipy.Integrate package in Python v.3.7.6. Kinetic parameters were estimated from literature, and initial conditions were set to experimental conditions, with intermediates species set at zero. Details of the ODE simulations are discussed in Supplementary Information.

Statistics and reproducibility. The number of replicates and types of replicates performed are described in the legend to each figure. Individual data points are shown, and where relevant, the average \pm s.d. is shown; this information is provided in each figure legend. The type of statistical analysis performed in Fig. $3 \mathrm{~d}$ and Extended Data Fig. 3 is described in the legend to each figure. Exact $P$ values along with degrees of freedom computed from the statistical analysis can be found in the source data.
Reporting Summary. Further information on research design is available in the Nature Research Reporting Summary linked to this article.

\section{Data availability}

All data presented in this paper are available as source data and as supplementary data. All source data as well as Supplementary Data 2 and 3 are also deposited in Mendeley Data (doi: $10.17632 / \mathrm{hr} 3 j 3 y z t x b .1)^{57}$. All plasmids used in this paper are available in Addgene with the identifiers 140371, 140374, 140391 and 140395. Source data are provided with this paper.

\section{Code availability}

The Jupyter Notebook files with the Python codes used in Extended Data Fig. 5, Extended Data Fig. 8 and Fig. 6 are provided as Supplementary Data 6, and the ODE model used in this paper is described in the Supplementary Information. The Python codes are also available in GitHub at https://git.io/Jtlh1(ref. ${ }^{58}$ ).

\section{References}

56. Ohgane, K. \& Yoshioka, H. Quantification of gel bands by an Image J macro, band/peak quantification tool. protocols.io https://doi.org/10.17504/protocols. io 7 vghn $3 \mathrm{w}$ (2019).

57. Jung, J. K., Archuleta, C. M., Alam, K. K. \& Lucks, J. B. Programming cell-free biosensors with DNA strand displacement circuits. Mendeley Data V2 https://doi.org/10.17632/hr3j3yztxb.2 (2021).

58. Jung, J. K., Archuleta, C. M., Alam, K. K. Lucks, J. B. Programming cell-free biosensors with DNA strand displacement circuits. GitHub https://git.io/Jtlh1 (2021).

\section{Acknowledgements}

We thank A. Thompson (Northwestern University) and C. Knopp (Northwestern University) for managing the experimental reagents and equipment used in this study; J. Hester (Northwestern University), A. Curtiss (Northwestern University) and J. Mamish (Northwestern University) for helpful discussion on the ADC circuit architecture; S. Schaffter (National Institute of Standards and Technology) for manuscript editing and helpful discussion on TMSD circuits; J. Peruzzi (Northwestern University) for assistance with NanoDrop measurements; S. Pshenychnyi (Recombinant Protein Production Core at Northwestern University) for assistance in protein purification. J.K.J. was supported in part by Northwestern University's Graduate School Cluster in Biotechnology, System, and Synthetic Biology, which is affiliated with the Biotechnology Training Program, by a Ryan Fellowship and by a McCormick School of Engineering Terminal Year Fellowship. C.M.A. was supported by the Department of Defense through the National Defense Science \& Engineering Graduate (NDSEG) Fellowship program. This work was also supported by funding from NSF CAREER (1452441 to J.B.L.), NSF MCB RAPID (1929912 to J.B.L.), support from the Crown Family Center for Jewish and Israel Studies at Northwestern University (to J.B.L.) and Searle Funds at The Chicago Community Trust (to J.B.L.).

\section{Author contributions}

J.K.J., K.K.A. and J.B.L. designed the study. J.K.J., C.M.A. and J.B.L. analyzed the data. J.K.J., C.M.A. and K.K.A. conducted the research. J.K.J., K.K.A. and J.B.L. developed the methodology. J.K.J. and J.B.L. undertook visualization of the data. J.K.J. and C.M.A. developed the software. J.K.J., K.K.A., C.M.A. and J.B.L. wrote the article. J.K.J. and J.B.L. curated the data. J.B.L. acquired funding for the study. J.K.J. and C.M.A. validated the results. J.K.J. and J.B.L. managed and coordinated the study. J.K.J. and J.B.L. supervised the research.

\section{Competing interests}

K.K.A., J.K.J. and J.B.L. have submitted an international patent application that has been nationalized in the USA (No. US 17/309,240) and in Europe (No. EP19881824.7) relating to regulated in vitro transcription reactions, an international patent application (PCT/US2020/030112, No. 62/838,852) relating to the preservation and stabilization of in vitro transcription reactions, and a US provisional application (PCT/US2020/030112 or US provisional No. 63/154,247) relating to cell-free biosensors with DNA strand displacement circuits. K.K.A. and J.B.L. are founders and have financial interest in Stemloop, Inc. The latter interests are reviewed and managed by Northwestern University in accordance with their conflict of interest policies. C.M.A. declares no competing interests.

\section{Additional information}

Extended data is available for this paper at https://doi.org/10.1038/s41589-021-00962-9.

Supplementary information The online version contains supplementary material available at https://doi.org/10.1038/s41589-021-00962-9.

Correspondence and requests for materials should be addressed to Julius B. Lucks.

Peer review information Nature Chemical Biology thanks Jerome Bonnet and the other, anonymous, reviewer(s) for their contribution to the peer review of this work.

Reprints and permissions information is available at www.nature.com/reprints. 

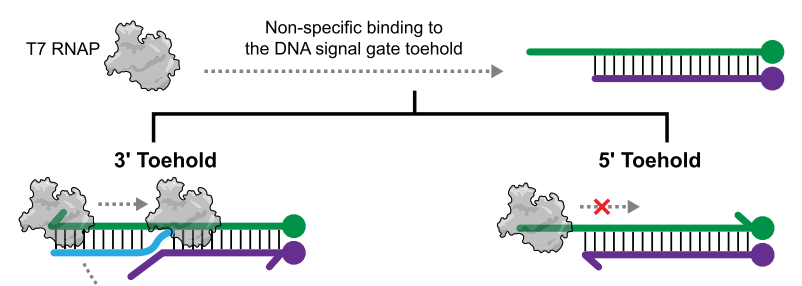

RNA side product

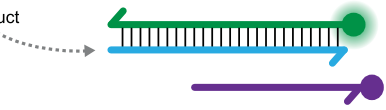

b

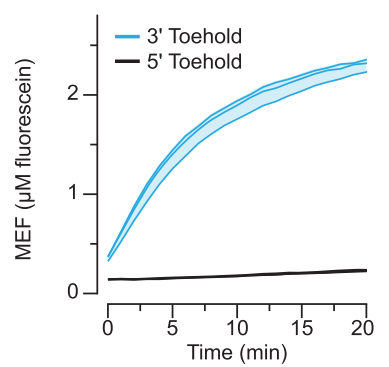

c

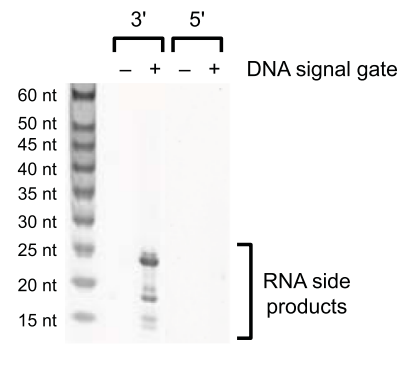

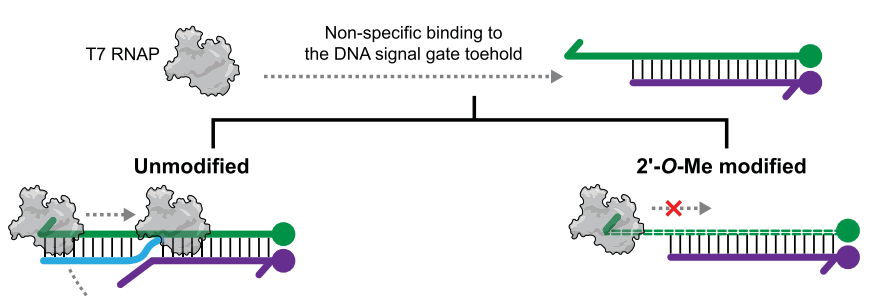

e

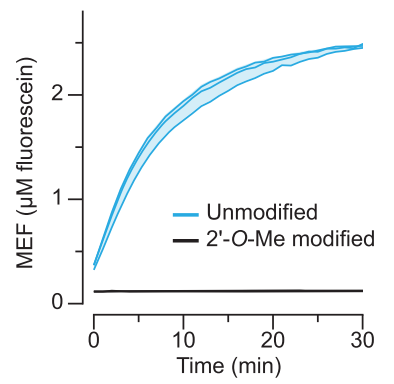

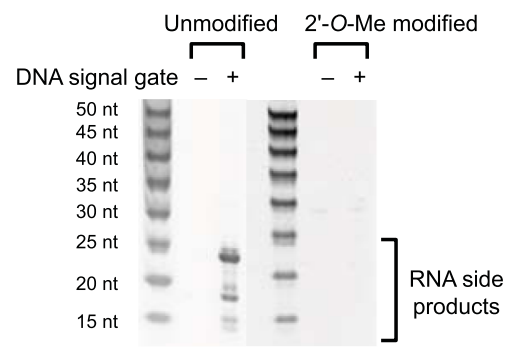

Extended Data Fig. 1 | Toehold-mediated DNA strand displacement can be used to track RNA outputs with an appropriately designed DNA gate. a, In the presence of IVT components, T7 RNAP can non-specifically bind to the toehold region of the DNA signal gate. When the toehold is on the $3^{\prime}$ end of the gate, this leads to transcription of unwanted RNA side products that can displace the quencher strand. This process is blocked when the toehold is on the $5^{\prime}$ end of the gate. $\mathbf{b}$, The $3^{\prime}$ toehold DNA signal gate leads to fluorescence activation in the presence of T7 RNAP, while the 5' toehold DNA signal gate does not. c, When the reaction products from $\mathbf{b}$ were extracted and run on an urea-polyacrylamide gel, RNA side products appear only from the $3^{\prime}$ toehold DNA signal gate. A negative control where no DNA signal gate is present in the reaction (-) was run alongside for both $3^{\prime}$ and $5^{\prime}$ toeholds. d, Modifying the DNA gate with 2'-O-methyl oligonucleotides prevents promoter-independent transcription by T7 RNAP. e, When the 3' toehold DNA signal gate is modified with 2'-O-methyl oligonucleotides, no fluorescence activation is observed in the absence of a T7 RNAP-driven IVT template. f, When the reactions from e were run on an urea- polyacrylamide gel, no RNA side products were observed from the $2^{\prime}$-O-methyl DNA signal gate. A negative control where no DNA signal gate is present in the reaction (-) was run alongside for both unmodified and modified gates. Data shown in $\mathbf{b}$ and $\mathbf{e}$ are $n=3$ independent biological replicates each plotted as a line with raw fluorescence standardized to MEF ( $\mu$ M fluorescein). Shading indicates the average of the replicates \pm standard deviation. Data shown in $\mathbf{c}$ and $\mathbf{f}$ are a representative of $n=3$ independent biological replicates. The uncropped, unprocessed gel image shown in $\mathbf{c}$ and $\mathbf{f}$ is available as Source Data. 

Variant 1: GGCUCGAG... Variant 2: GGUGCUCG...
Variant 2 Strengthened: GGAUCUCG... Variant 3: GGUAACUC... Variant 3 Strengthened: GGGAUCUC...

b

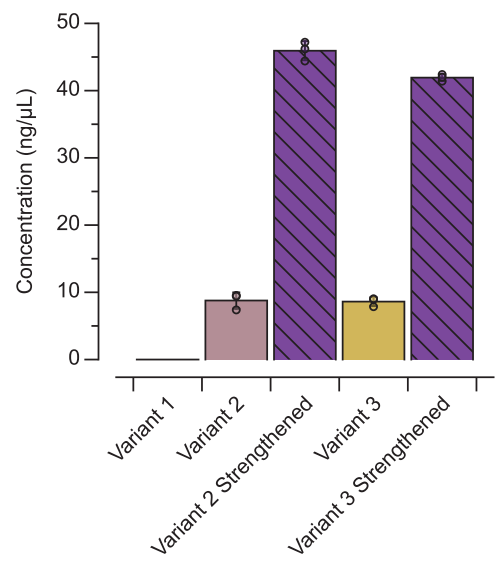

C

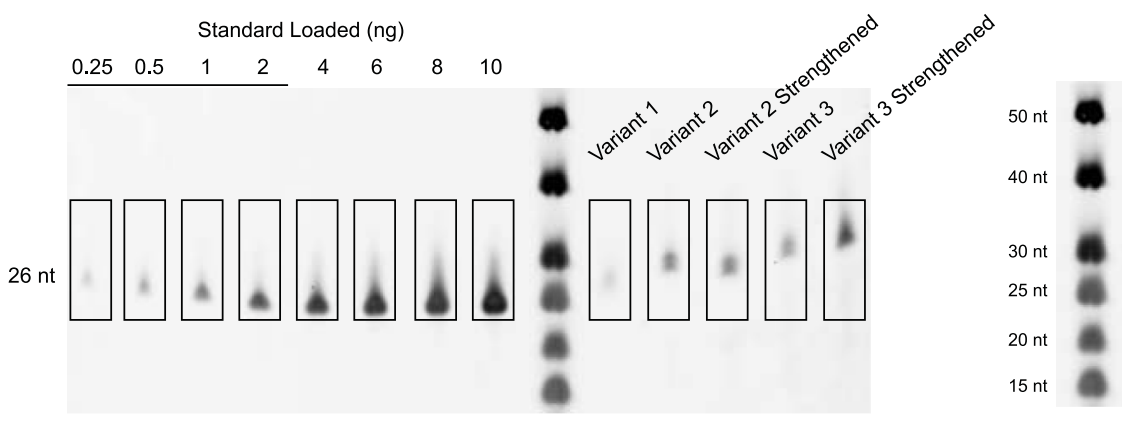

d

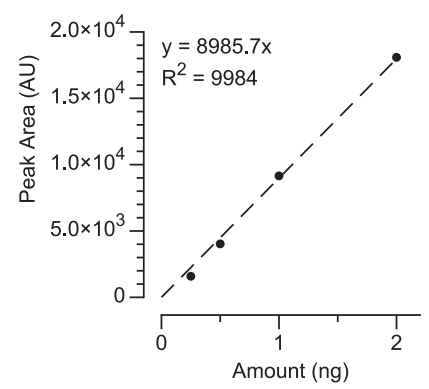

e

\begin{tabular}{|r|r|r|r|}
\multicolumn{3}{c}{ Amount (ng) } \\
\hline RNA & Peak Area & PAGE & Qubit \\
\hline Variant 1 & 2640.134 & 0.29 & N/A \\
Variant 2 & 9404.631 & 1.05 & 0.954 \\
Variant 2 Strengthened & 10291.167 & 1.15 & 4.72 \\
Variant 3 & 7431.388 & 0.83 & 0.896 \\
Variant 3 Strengthened & 20183.078 & 2.25 & 4.24 \\
\hline
\end{tabular}

Extended Data Fig. 2 | Transcription efficiency impacts the speed of TMSD. a, The eight initially transcribed nucleotides of each InvadeR variant in Fig. 2a. Nucleotides that are not part of the InvadeR sequences are bolded, and inserted nucleotides are underlined. $\mathbf{b}$, Concentrations of each variant from IVT reactions measured by the Qubit RNA HS assay kit (Invitrogen, catalog no. Q32852). Each variant was produced in situ in the presence of the DNA signal gate for 30 min and extracted (see the RNA extraction from IVT reactions section in Materials and Methods). The concentration of variant 1 was too low for Qubit quantification. $\mathbf{c}$, The samples measured in $\mathbf{b}$ were run on an urea-polyacrylamide gel and stained with SYBR gold. Titration of an RNA standard of a similar length was performed to determine the linear range of band peak area quantified by Fiji-Image ${ }^{56}$. $\mathbf{d}, \mathrm{A}$ calibration curve was constructed by plotting the peak area computed from Fiji-ImageJ quantification against the total amount of standard loaded. e, Using the calibration curve in d, the total amount of RNA for each variant was determined and compared to the measurements made by Qubit in $\mathbf{b}$. Data in $\mathbf{b}$ are shown for $n=3$ independent biological replicates each plotted as a point with the bar height representing the average. Error bars indicate the average of the replicates \pm standard deviation. Data shown in $\mathbf{c}$ are a representative of $n=3$ independent biological replicates. The uncropped, unprocessed gel image shown in $\mathbf{c}$ is available as Source Data. 
GG-tetO-InvadeR

e TetR Family (+) 50 nM DNA template

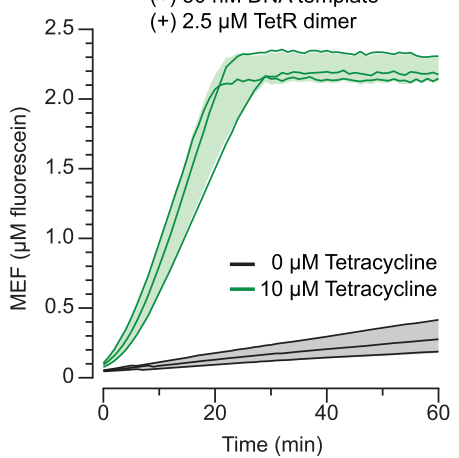

g

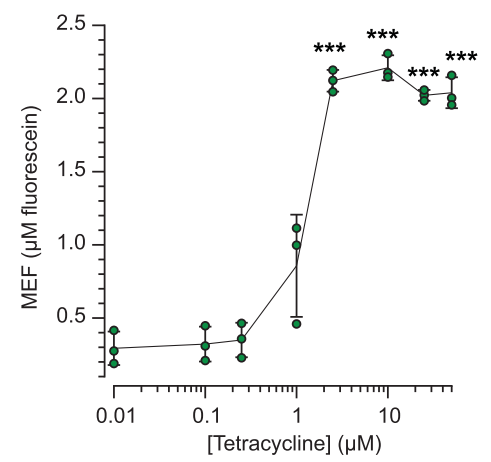

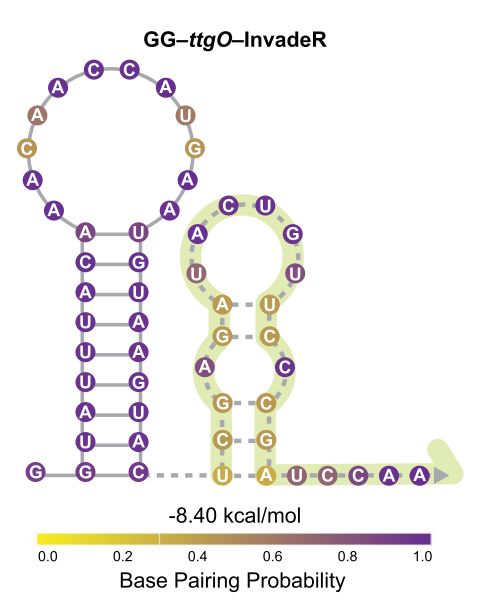

b

MarR Family

(+) 10 nM DNA template

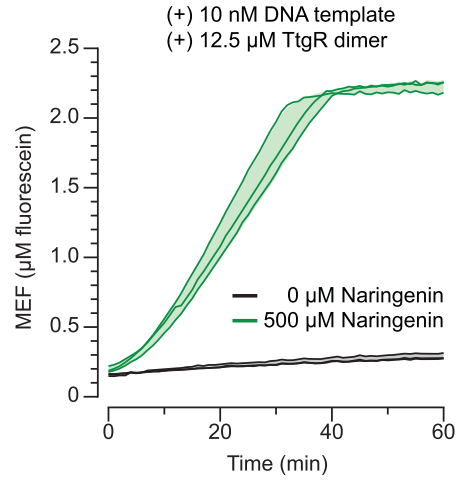

h

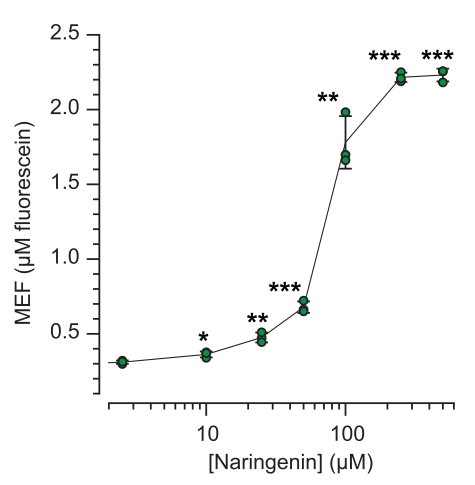

c

GA-smtO-Hairpin2-InvadeR (1BP Spacer)

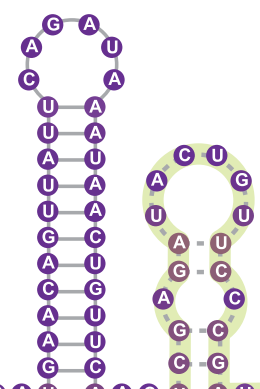

$\triangle A O A O A D-A D O C-A O C O A A$

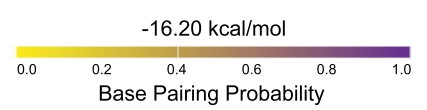

f

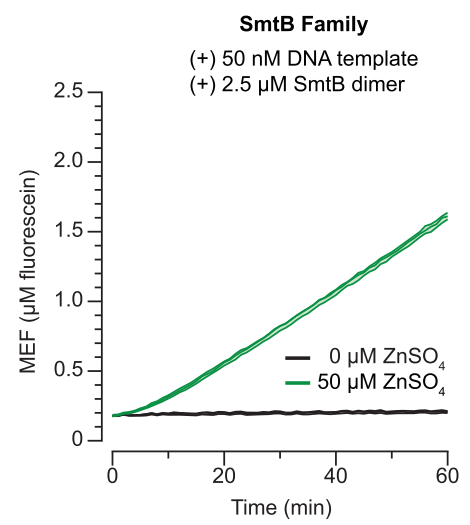

i

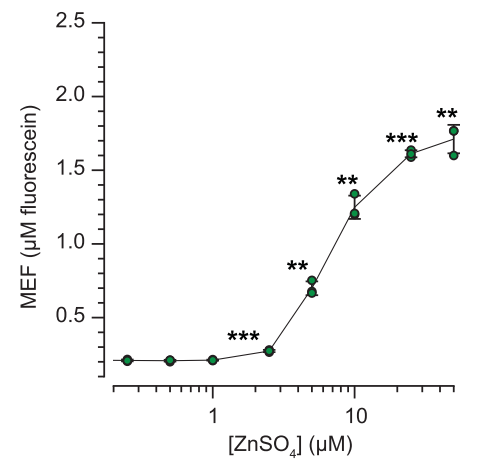

Extended Data Fig. 3 | Different input molecules can be detected by modularly configuring InvadeR with an aTF operator sequence. DNA templates encoding InvadeR are modified to contain the T7 promoter followed by a 2-bp spacer and an aTF operator sequence upstream of the InvadeR sequence. Secondary structures, minimum free energies and base pairing probabilities of $\mathbf{a}$, GG-tetO-InvadeR, b, GG-ttgO-InvadeR and $\mathbf{c}$, GA-smtO-Hairpin2InvadeR (1BP spacer variant) are predicted using NUPACK at $37^{\circ} \mathrm{C}^{30}$. The GA-smtO-Hairpin2-InvadeR sequence includes a hairpin designed to minimize structural interference of InvadeR by smtO (Extended Data Fig. 4h, i). The sequence complementary to the signal gate is denoted with a dotted line and highlighted in green shading. $\mathbf{d}$, TetR is used to sense tetracycline. e, TtgR, a MarR-family aTF, is used to sense naringenin. $\mathbf{f}$, SmtB is used to sense zinc. Variations in activation kinetics match the trend where the predicted secondary structure of InvadeR impacts the induction speed. To evaluate the limit of detection of each sensor, a dose response curve was created for $\mathbf{g}$, tetracycline, $\mathbf{h}$, naringenin and $\mathbf{i}, \mathrm{ZnSO}_{4}$ ( 1 h end-point data). All data shown are $\mathrm{n}=3$ independent biological replicates each plotted as a line (d-f) or a point ( $(\mathbf{g}-\mathbf{i})$ with raw fluorescence values standardized to MEF ( $\mu \mathrm{M}$ fluorescein). Shading (d-f) and error bars $(\mathbf{g}-\mathbf{i})$ indicate the average of the replicates \pm standard deviation. The ligand concentrations at which the signals are distinguishable from the background were determined using a two-sided, heteroscedastic Student's t-test against the no-ligand condition, and their $P$ value ranges are indicated with asterisks ( ${ }^{\star \star \star} P<0.001,{ }^{\star \star} P=0.001-0.01,{ }^{\star} P=0.01-0.05$ ). Exact $P$ values along with degrees of freedom can be found in Source Data. Data for the no ligand condition were excluded because the $x$-axis is on the log scale and are presented in Source Data. 


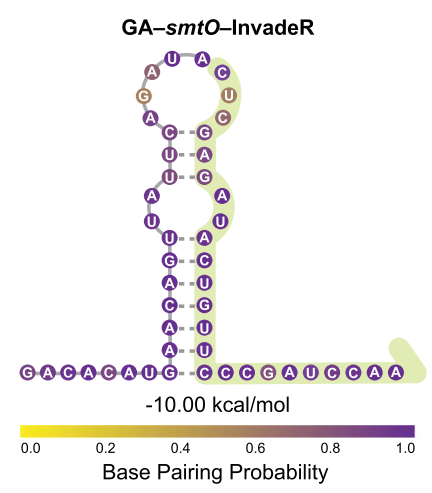

d

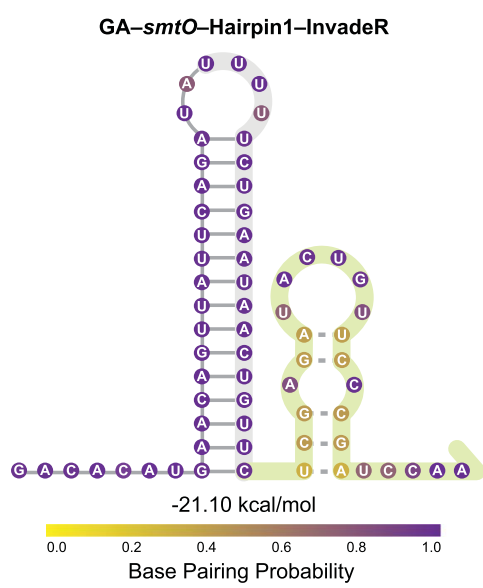

g

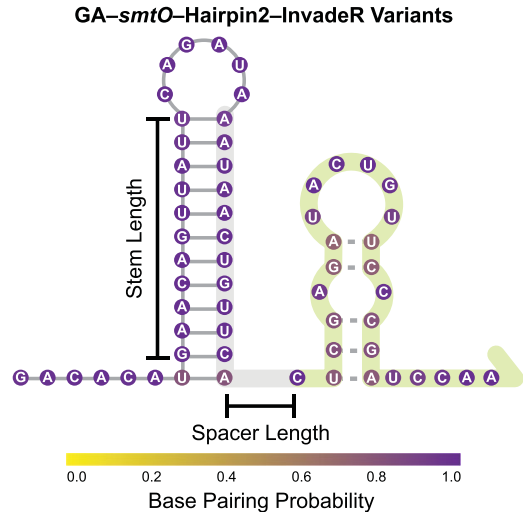

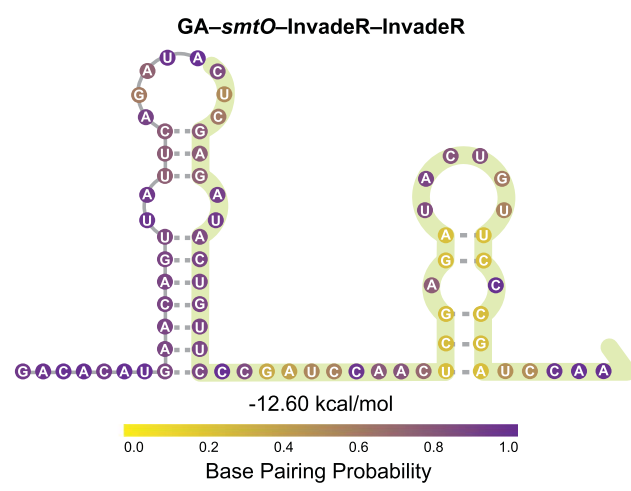

e

GA-smtO-Hairpin2-InvadeR

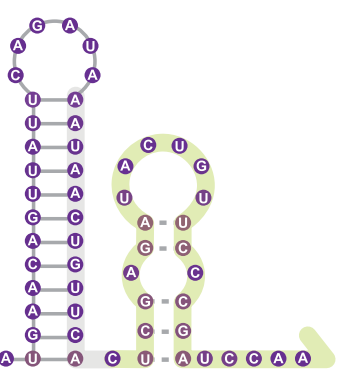

$-15.80 \mathrm{kcal} / \mathrm{mol}$

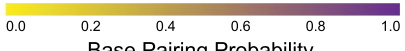

h

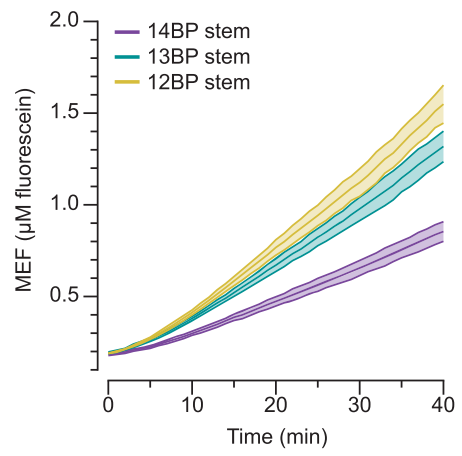

c

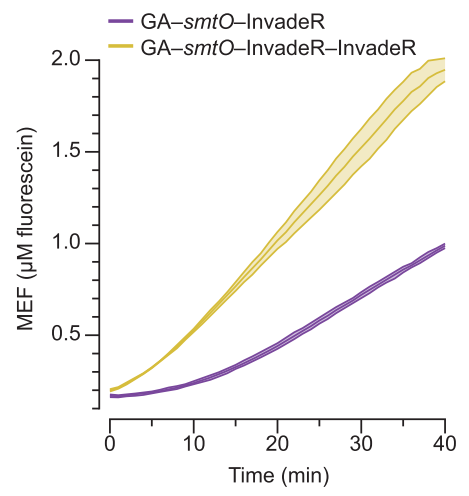

f

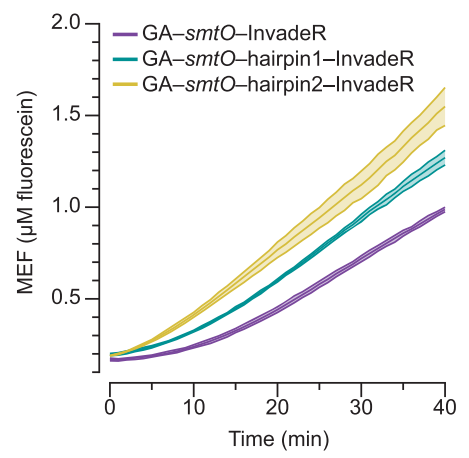

i

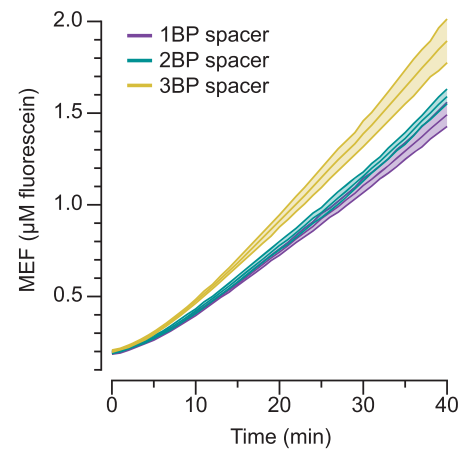

Extended Data Fig. 4 | Extra nucleotides can be added between the operator and InvadeR sequence to tune the kinetics of the zinc sensor. a, Secondary structure, minimum free energy and base pairing probabilities of GA-smtO-InvadeR predicted by NUPACK at $37^{\circ} \mathrm{C}^{30}$. A part of the wild type smtO sequence forms a strong predicted stem-loop with InvadeR. The nucleotides highlighted in green correspond to the InvadeR sequence that strand-displaces the DNA signal gate. $\mathbf{b}$, Secondary structure, minimum free energy and base pairing probabilities of GA-smtO-InvadeR-InvadeR predicted by NUPACK at $37^{\circ} \mathrm{C}^{30}$. c. Comparison of the kinetics of the unregulated GA-smtO-InvadeR reaction and the unregulated GA-smtO-InvadeR-InvadeR reaction. $\mathbf{d}, \mathbf{e}$, NUPACK-predicted secondary structures, minimum free energies and base pairing probabilities of the two GA-smtO-Hairpin-InvadeR variants designed to sequester the $s m t O$ sequence and prevent it from binding to InvadeR. The nucleotides highlighted in grey indicate the added sequestering sequence. $\mathbf{f}$, Comparison of the kinetics of the unregulated reactions of the variants shown in d and e. g, The GA-smtO-Hairpin2-InvadeR variants were built by lengthening either the stem length or the spacer between the hairpin and the InvadeR sequence. The stem length variants are built with the 0 -nt spacer, and the spacer variants are built with the 12-bp stem length. $\mathbf{h}, \mathbf{i}$, Comparison of the kinetics of the unregulated reactions of the GA-smtO-Hairpin2-InvadeR variants. All data shown are $n=3$ independent biological replicates each plotted as a line with raw fluorescence values standardized to MEF ( $\mu \mathrm{M}$ fluorescein). Shadings indicate the average of the replicates \pm standard deviation. 
a

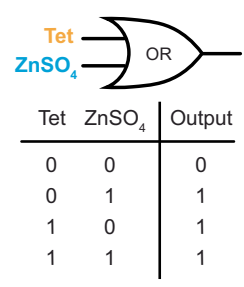

c

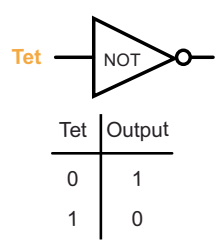

e

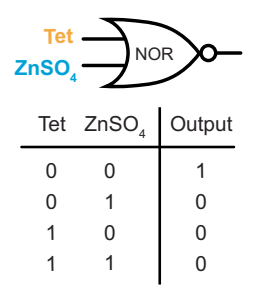

g

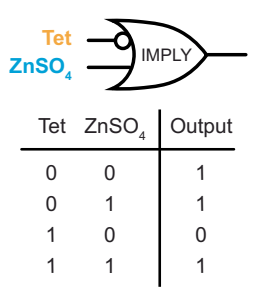

i

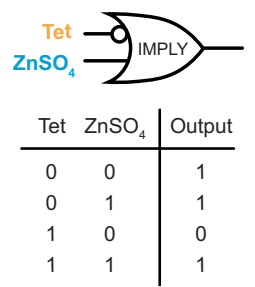

k

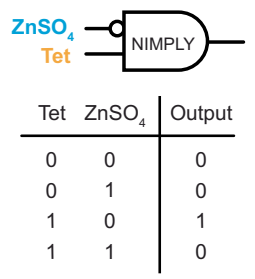

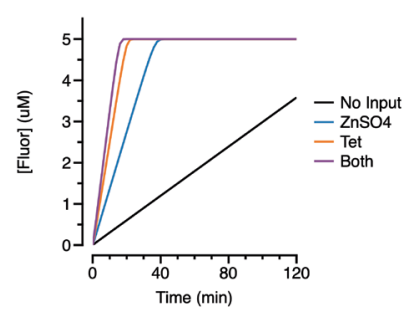
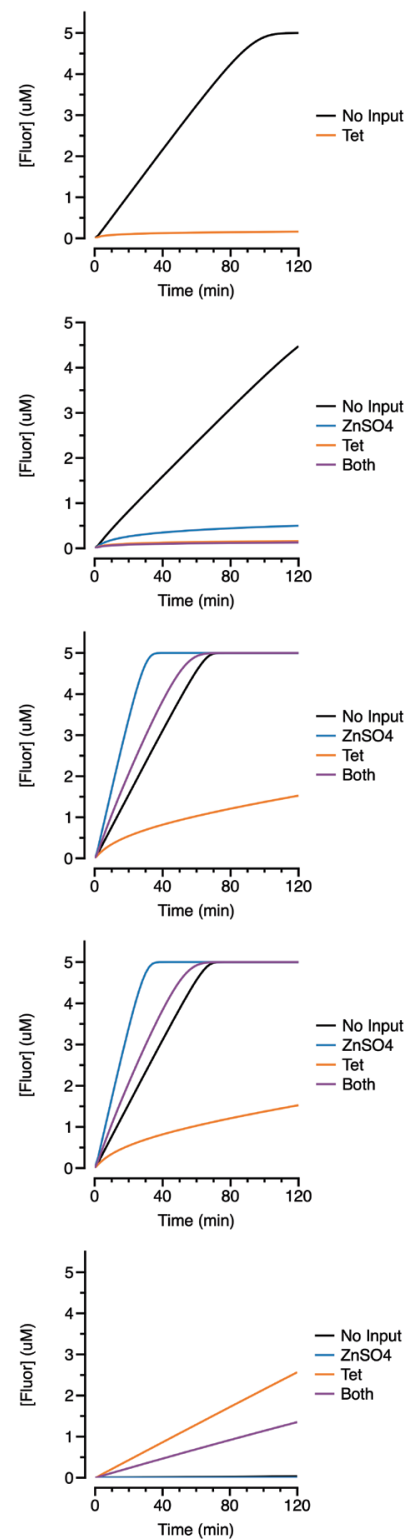

b

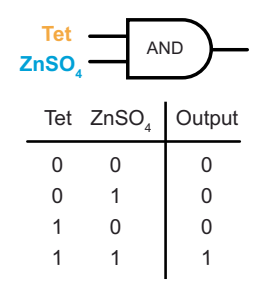

d
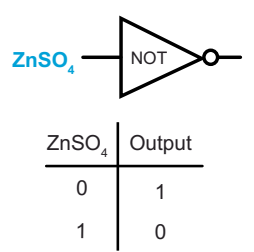

f

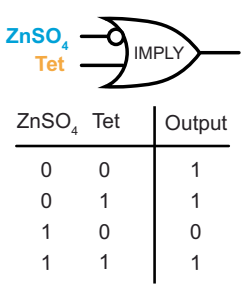

h

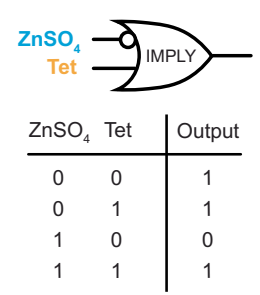

j

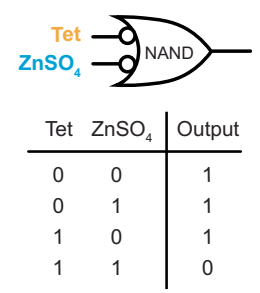

I

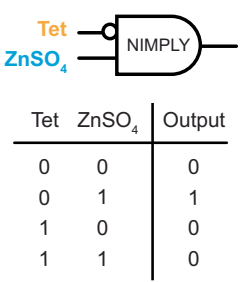

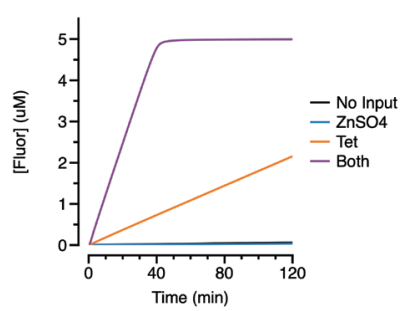
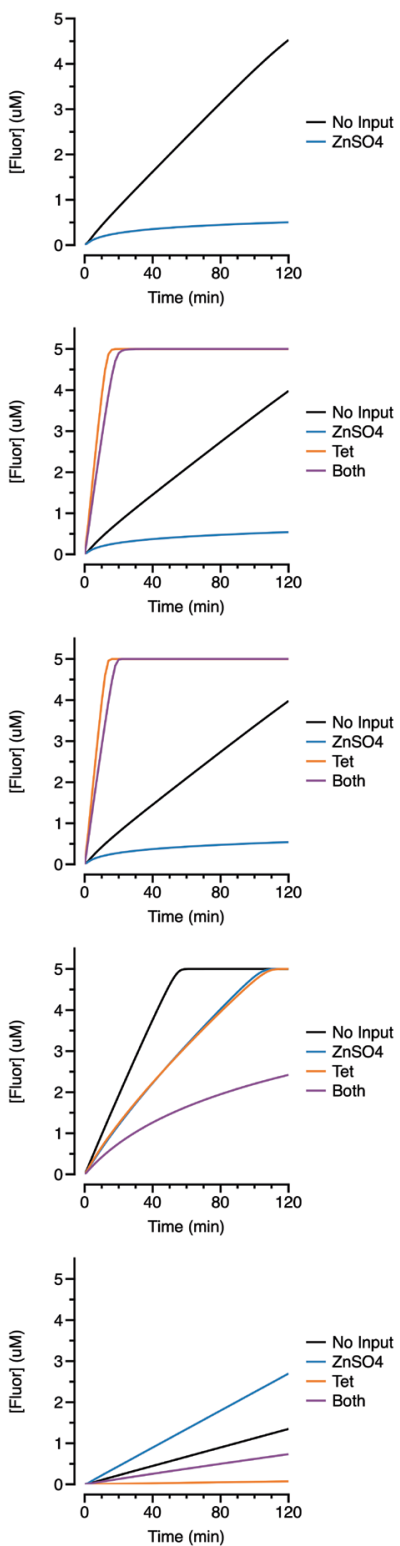

Extended Data Fig. 5 | ODE Modeling of Logic Gates. Simulations of logic gates discussed in Figs. 4, 5 and Extended Data Fig. 7 are shown along with the expected simulated trajectory trends. All Jupyter notebook codes used to simulate the results are available as Supplementary Data 6, and the method used to develop the ODE model for each representative logic gate is discussed in the Supplementary Information. 

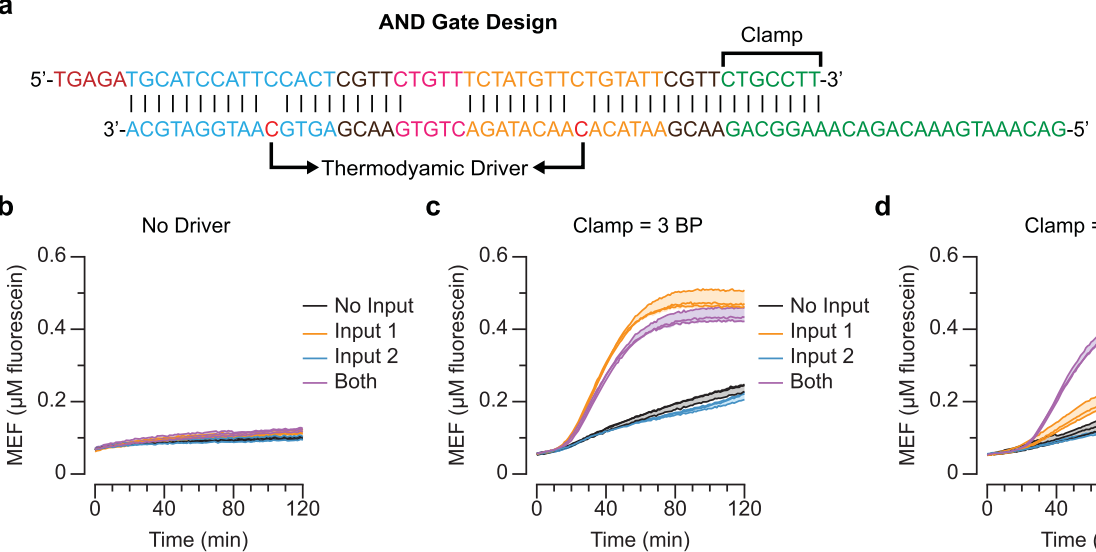

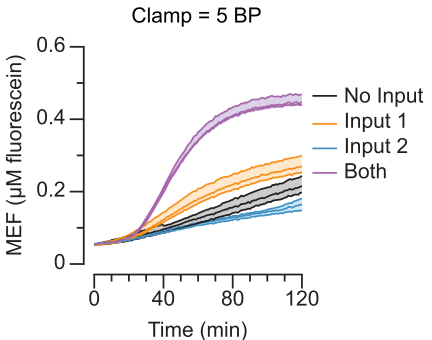

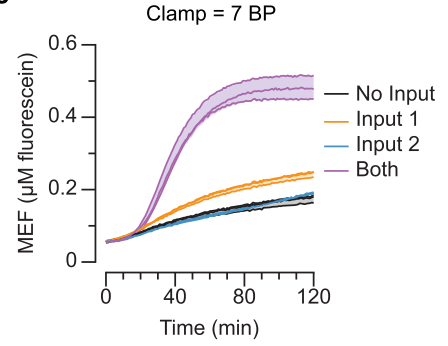

f

3'-GCAAGACGGAAAAAGACAAAGTAAACAGGG-5' InvadeR Mismatch Variant 1

5'-GGCGUUCUGCCUUUUUCUGUUUCAUUUGUCUU

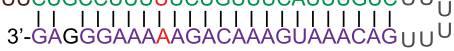
NOT Gate Design 1

[NOT Gate 1] + InvadeR 1

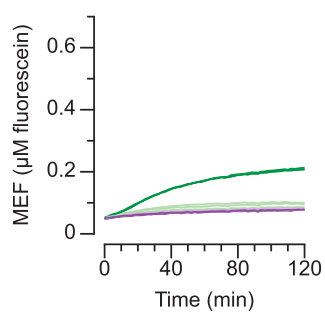

[NOT Gate 1] + InvadeR 2

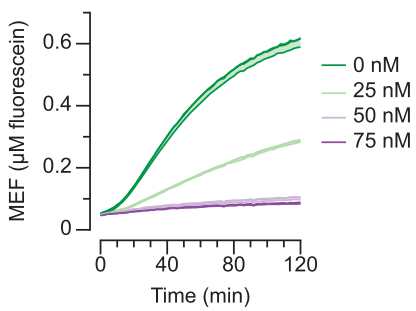

InvadeR Mismatch Variant 2

政

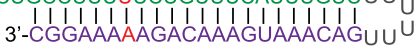
NOT Gate Design 2

5'-GGACGUUCUGCCUUUUUCUGUUUCAUUUGUCUU

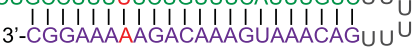
NOT Gate Design 3 i

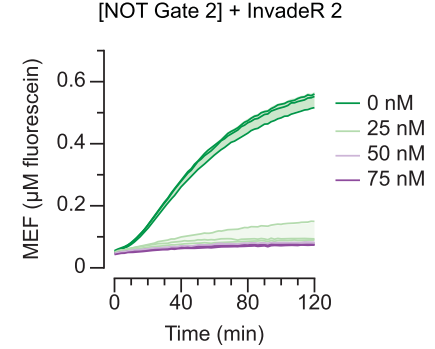

I

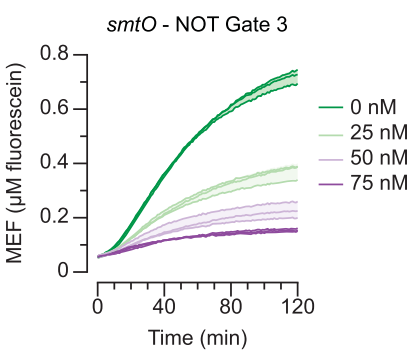

[NOT Gate 3] + InvadeR 2
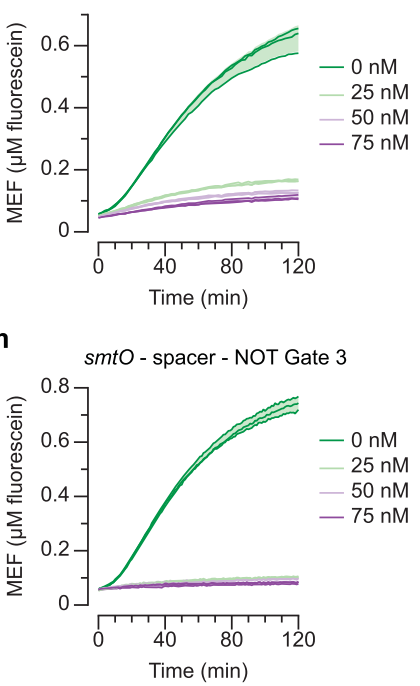

Extended Data Fig. 6 | Design Features of Basic Logic Gate Components. a, The sequence and design of the DNA AND gate shown in Fig. 4c. The mismatches act as the thermodynamic driver to run the reaction forward. The clamp domain prevents the top strand from being strand-displaced only with Input 1 (orange). b. Without thermodynamic drivers, no signal is observed. Lengthening the clamp reduces the leak by Input 1 . The leak observed from a c, 3-bp, d, 5-bp and e, 7-bp clamp. f, The sequences and designs of InvadeR and RNA NOT gates without any operator sequences. The mismatch between InvadeR and the signal gate is highlighted in red. Variant 2 incorporates 6 extra adenines that strengthen its interactions with the NOT gate. NOT gate 1 has a 4-nt toehold while NOT gate 2 and 3 have 6-nt toeholds. NOT gate 3 has an extra adenine following the initiating guanines that increases its transcription efficiency ${ }^{36}$. Titration of the template encoding NOT gate 1 in the presence of 25 nM of the template encoding InvadeR $\mathbf{g}$, variant 1 and $\mathbf{h}$, variant 2 . While variant 2 requires more of the NOT gate template to block signal, stronger signal is observed from variant 2 without the NOT gate. Titration of the template encoding NOT gate $\mathbf{i}, 2$ and $\mathbf{j}, 3$ in the presence of $25 \mathrm{nM}$ of the InvadeR variant 2 template. NOT gate 2 and 3 are more efficient at sequestering InvadeR than NOT gate 1. k, Incorporating the operator sequence into the NOT gate impacts its structure. NUPACK predicts that the smtO sequence (light grey) interacts with a part of NOT gate 3, blocking the toehold ${ }^{30}$. A spacer sequence (underlined) can restore the expected structure of the NOT gate. Titration of the template encoding I, smtO-NOT gate 3 and $\mathbf{k}$, smtO-spacer-NOT gate 3 in the presence of 25 nM of the InvadeR variant 2 template. All reactions shown are unregulated. All data shown are $n=3$ independent biological replicates each plotted as a line with raw fluorescence standardized to MEF ( $\mu \mathrm{M}$ fluorescein). Shading indicates the average of the replicates \pm standard deviation. 
a

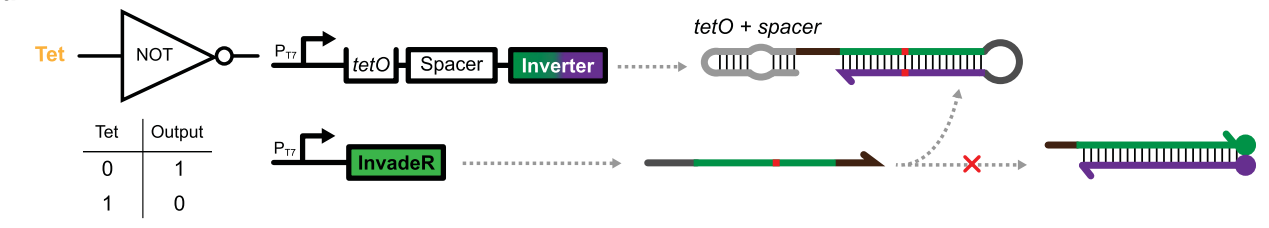

c

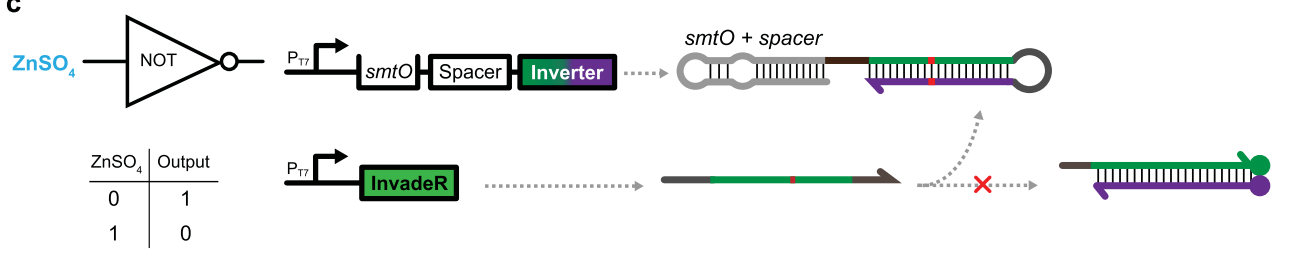

e
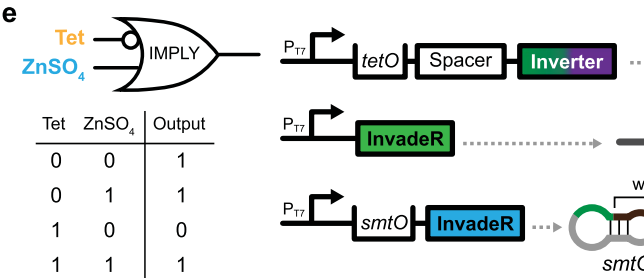

tetO + spacer

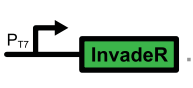

PII $\longrightarrow$ smto InvadeR

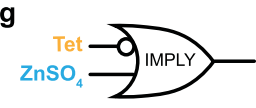
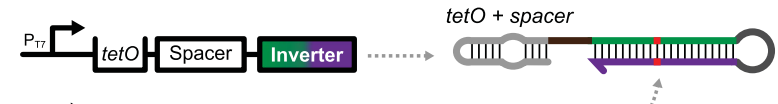

\begin{tabular}{cc|c} 
Tet & $\mathrm{ZnSO}_{4}$ & Output \\
\hline 0 & 0 & 1
\end{tabular}

\begin{tabular}{ll|l}
0 & 1 & 1
\end{tabular}

\begin{tabular}{ll|l}
1 & 0 & 0
\end{tabular}

PrT

\begin{tabular}{ll|l}
1 & 1 & 1
\end{tabular}

$\mathrm{P}_{\mathrm{Tr}} \mathrm{Ismto}_{\mathrm{InvadeR}}$
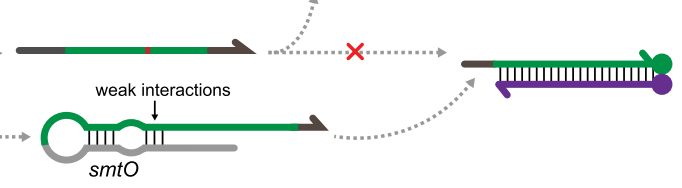

i
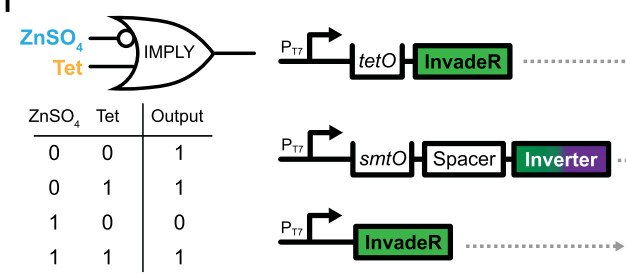

teto

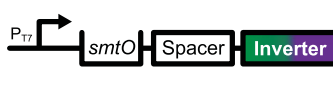

smto + spacer

Pri $\longrightarrow$ InvadeR

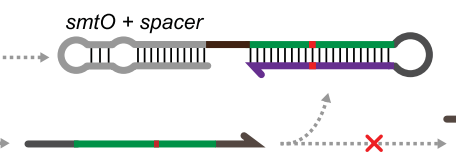

|| || || IIIIIIIIIIII-

k

$$
\begin{array}{cc|c}
\mathrm{ZnSO}_{4} & \multicolumn{2}{c}{\mathrm{Tet}_{\text {NIMPLY }}} \\
\mathrm{Tet} & \mathrm{ZnSO}_{4} & \text { Output } \\
\hline 0 & 0 & 0 \\
0 & 1 & 1 \\
1 & 0 & 0 \\
1 & 1 & 0
\end{array}
$$

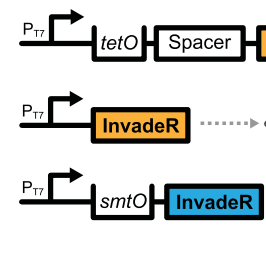

b

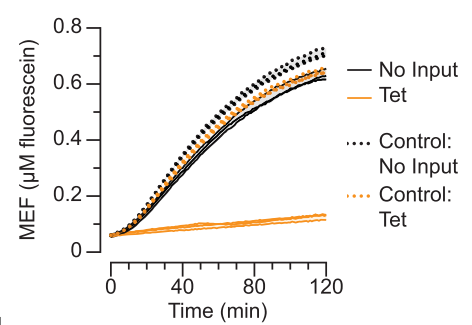

d

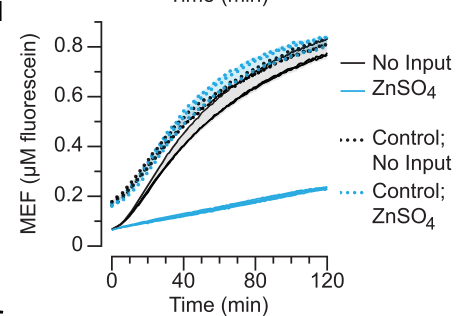

f

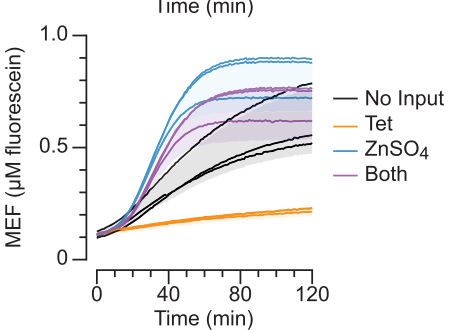

h

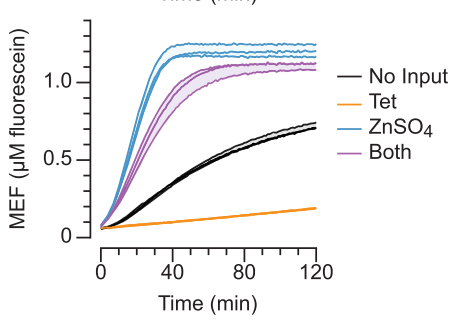

j
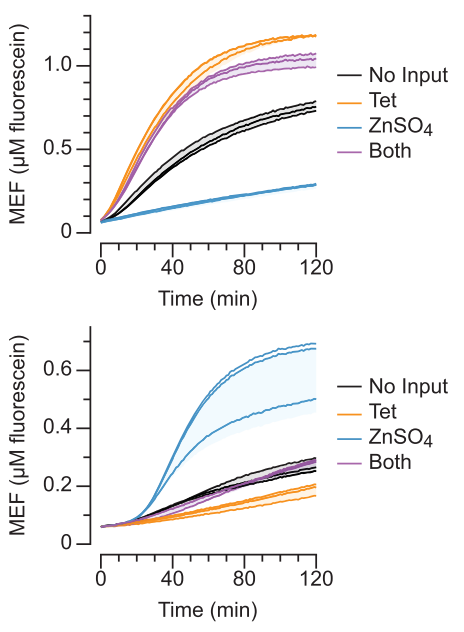

Extended Data Fig. 7 | Logic gates can be built modularly using ligand-induced RNA inputs. a, Tetracycline-induced NOT gate shown in Fig. 4e. b, A template encoding the shuffled sequence of the TetR-regulated NOT gate was used as a control to demonstrate that the tetracycline-induced signal reduction is not due to resource limitations. $\mathbf{c}, \mathrm{ZnSO}_{4}$-induced NOT gate can be designed the same way. The spacer sequence was added to prevent the smtO sequence from disrupting the NOT gate structure (Extended Data Fig. 6). d, In the presence of $\mathrm{ZnSO}_{4}$, the fluorescence signal is deactivated. Similarly, control reactions with a template encoding the shuffled sequence of the SmtB-regulated NOT gate are shown. e, A tet IMPLY ZnSO ${ }_{4}$ gate designed as described in Fig. 5c. f, Fluorescence activation is observed unless only tetracycline is added. $\mathbf{g}, \mathrm{A}$ tet IMPLY $\mathrm{ZnSO}_{4}$ gate can be alternatively built by including a $\mathrm{ZnSO}_{4}$-inducible template that interacts with the signal gate instead of the OR gate. $\mathbf{h}$, The alternative IMPLY gate performs the expected logic computation. A faster signal generation from the $\mathrm{ZnSO}_{4}$-induced conditions is observed since the $\mathrm{ZnSO}_{4}$-induced $\mathrm{RNA}$ strands directly perform TMSD on the signal gate. $\mathbf{i}$, The alternative approach to building the IMPLY gate can be applied to the $\mathrm{ZnSO}_{4} I M P L Y$ tet gate, and $\mathbf{j}$, the gate performs the expected logic computation. $\mathbf{k}$, A tet NIMPLY $\mathrm{ZnSO}_{4}$ gate is designed the same way as the $\mathrm{ZnSO}_{4} \mathrm{NIMPLY}$ tet gate shown in Fig. $5 \mathrm{~g}$. I, The tet NIMPLY $\mathrm{ZnSO}_{4}$ gate performs the expected logic computation. All the experimental conditions used in this figure can be found in Supplementary Data 5. All data shown are $n=3$ independent biological replicates each plotted as a line with raw fluorescence standardized to MEF ( $\mu M$ fluorescein). Shading indicates the average of the replicates \pm standard deviation. 
oX Threshold

$-2 \mu \mathrm{MnSO}_{4}-5 \mu \mathrm{MnSO}_{4}$

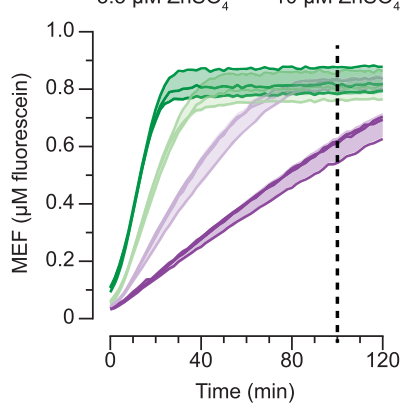

e

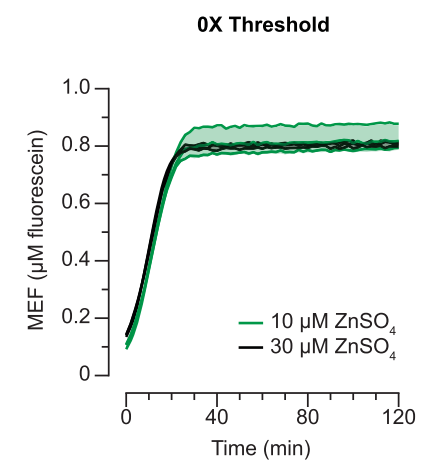

b

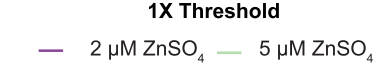
$-3.5 \mu \mathrm{M} \mathrm{ZnSO}_{4}-10 \mu \mathrm{M} \mathrm{ZnSO}_{4}$

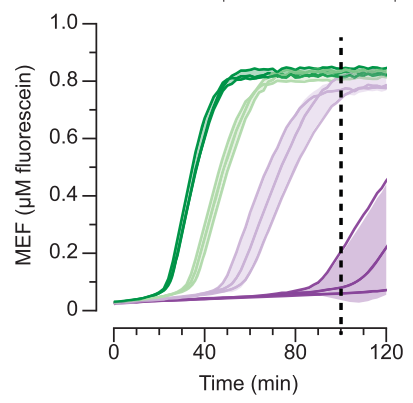

$\mathbf{f}$

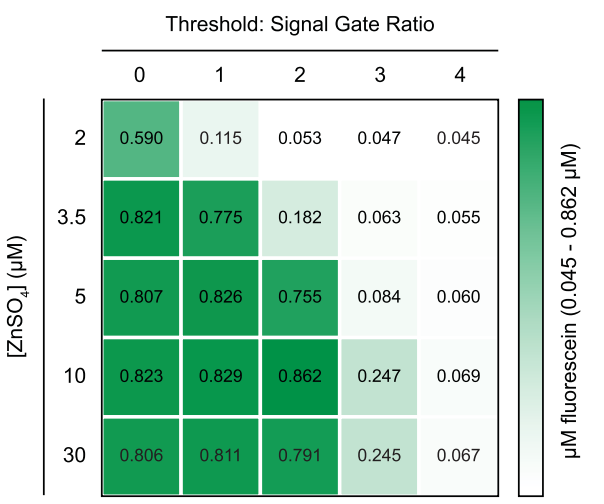

c

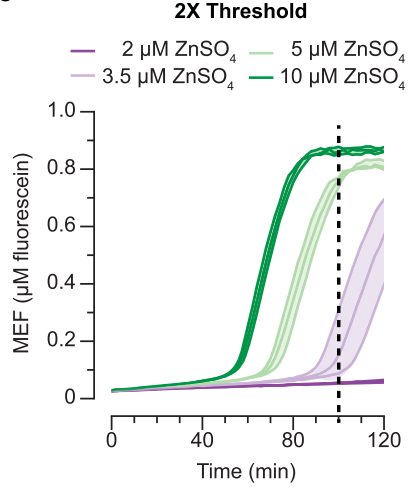

g

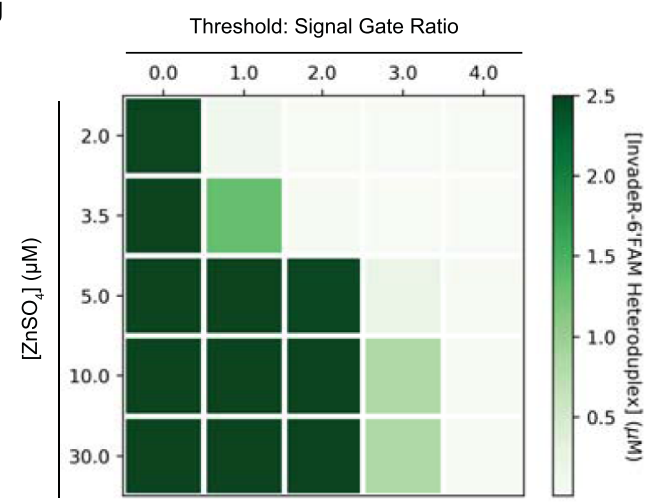

h

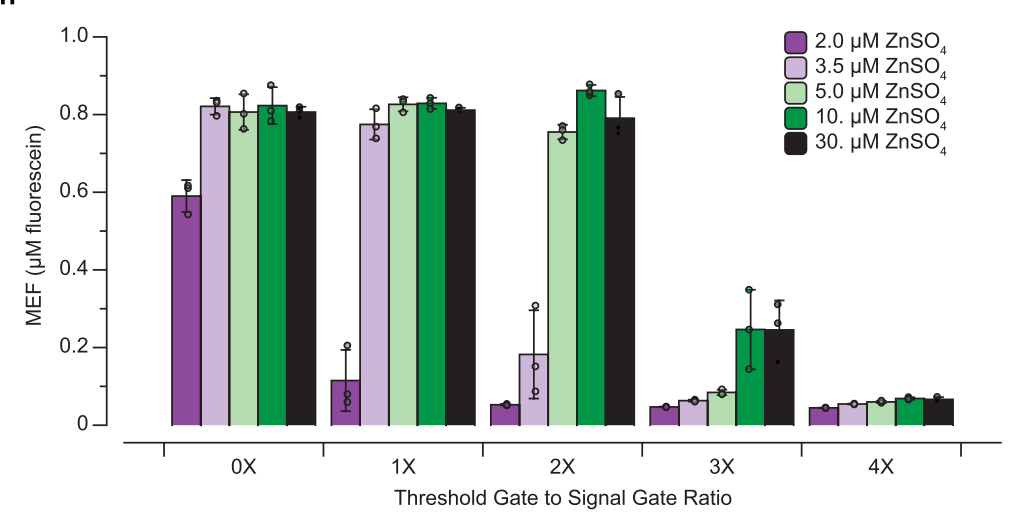

Extended Data Fig. 8 | The Semi-quantitative Standard Generated by the Genetic ADC Circuit Built with the Zinc Sensor. The kinetic traces corresponding to the data shown in Fig. 6 e are presented for the 8-nt threshold gate in different ratios above a fixed signal gate concentration (a, OX, $\mathbf{b}$, $1 X, \mathbf{c}, 2 X$ and $\mathbf{d}, 3 X$ threshold). The time point ( $t=100 \mathrm{~min}$ ) at which the heatmap was created is indicated with a vertical dotted line. The differences in the response speed for different zinc concentrations are essential for creating the standard. $\mathbf{e}$, The $10 \mu \mathrm{M}$ and $30 \mu \mathrm{M}$ zinc conditions show no kinetic differences without any threshold gate. $\mathbf{f}, \mathbf{g}$, The functional characterization and ODE predictions of the ADC circuit at $100 \mathrm{~min}$ from Fig. $6 \mathrm{e}, \mathrm{d}$, respectively, including the $30 \mu \mathrm{M}$ zinc and $4 \mathrm{X}$ threshold conditions. The $10 \mu \mathrm{M}$ and $30 \mu \mathrm{M}$ zinc conditions behave identically as predicted by the ODE model due to their identical kinetic behavior observed in $\mathbf{e}$. $\mathbf{h}$, The corresponding bar graph data of the semi-quantitative standard shown in $\mathbf{f}$. All data shown are $\mathrm{n}=3$ independent biological replicates each plotted as a line (a-e) or a point (h) with raw fluorescence values standardized to MEF ( $\mu \mathrm{M}$ fluorescein). The bars in $\mathbf{h}$ and the values on heatmap in $\mathbf{f}$ represent averages of the replicates. Shadings in a-e and error bars in $\mathbf{h}$ indicate the average of the replicates \pm standard deviation. 


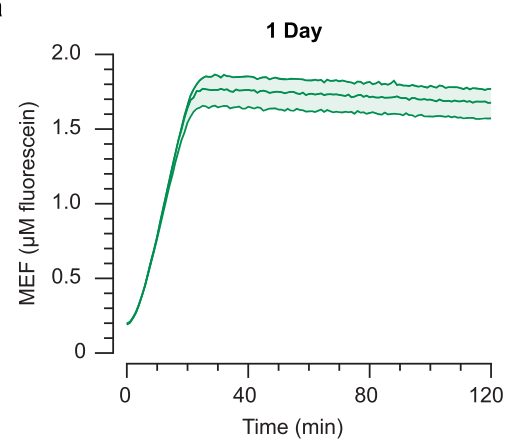

d

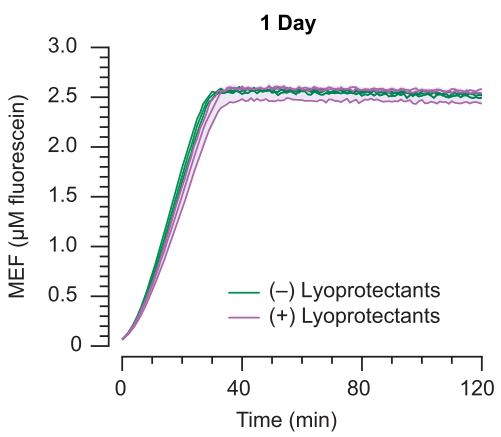

g

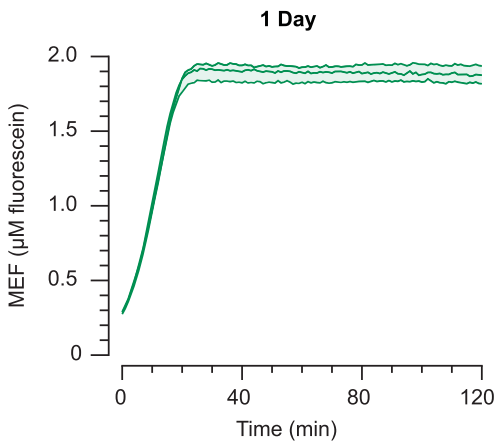

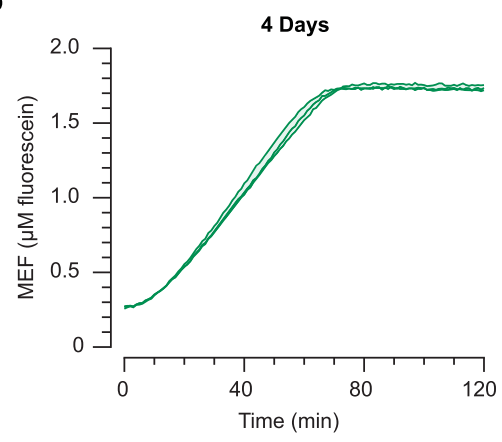

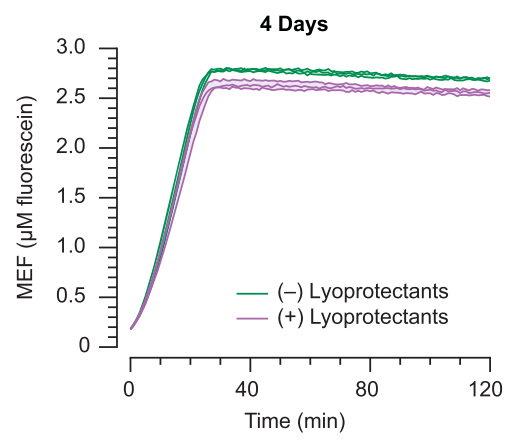

h

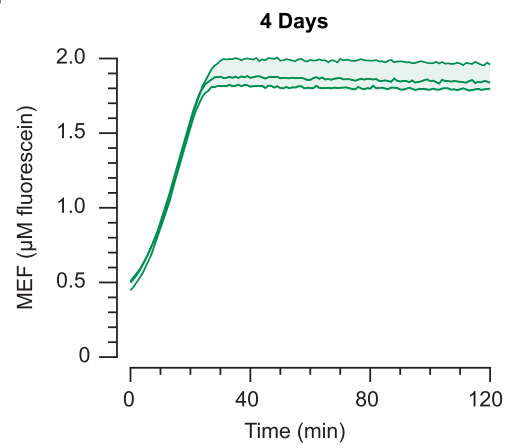

c

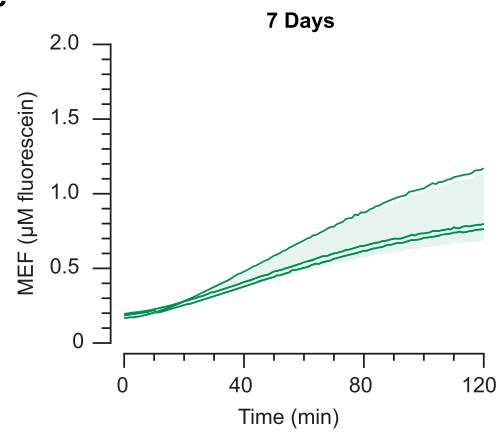

f

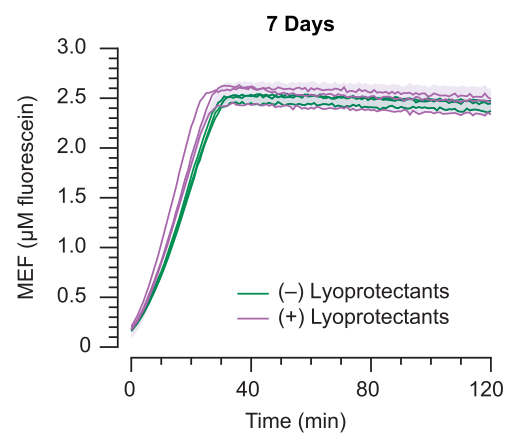

i

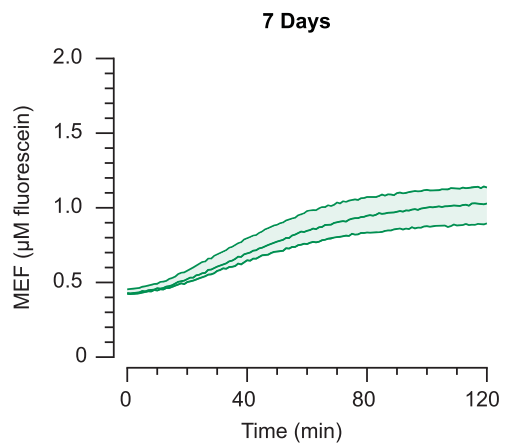

Extended Data Fig. 9 | ROSALIND with TMSD can be freeze-dried. Unregulated reactions were lyophilized overnight with the addition of $50 \mathrm{mM}$ sucrose and $250 \mathrm{mM} \mathrm{D}$-mannitol as the lyoprotectants unless otherwise indicated. The lyophilized reactions were then vacuum-packaged in a light protective bag with a dri-card and kept in a cool, shaded area until usage (see Materials and Methods for the detailed protocol). Kinetic traces of rehydrated reactions after $\mathbf{a}, 1$ day, $\mathbf{b}, 4$ days and $\mathbf{c}, 7$ days of storage are shown. There is a decrease in overall signal as well as in the response speed over time. To investigate the cause of the signal loss over time, the DNA signal gate alone was lyophilized overnight with or without the lyoprotectants, packaged and stored as described above. The DNA signal gate was rehydrated with the rest of the IVT components after $\mathbf{d}, 1$ day, e, 4 days and $\mathbf{f}, 7$ days. The response speed as well as the magnitude of the signal are maintained, indicating that the signal loss is likely due to instability of certain IVT components. To test this hypothesis, unregulated reactions with Tris-buffered NTPs, instead of NaOH-buffered NTPs, were lyophilized with the lyoprotectants, packaged and stored as described above. Kinetic traces of rehydrated reactions after $\mathbf{g}, 1$ day, $\mathbf{h}, 4$ days and $\mathbf{i}, 7$ days of storage are shown. The signal loss is somewhat mitigated with Tris-buffered NTPs, but a similar degree of signal loss is observed for a long-term storage of lyophilized reactions. All data shown are $n=3$ independent biological replicates each plotted as a line with raw fluorescence values standardized to MEF ( $\mu \mathrm{M}$ fluorescein). Shadings indicate the average of the replicates \pm standard deviation. 


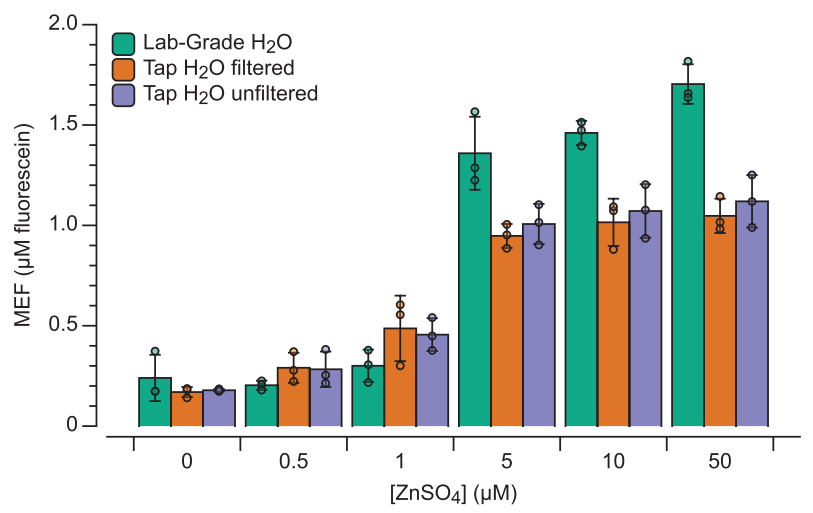

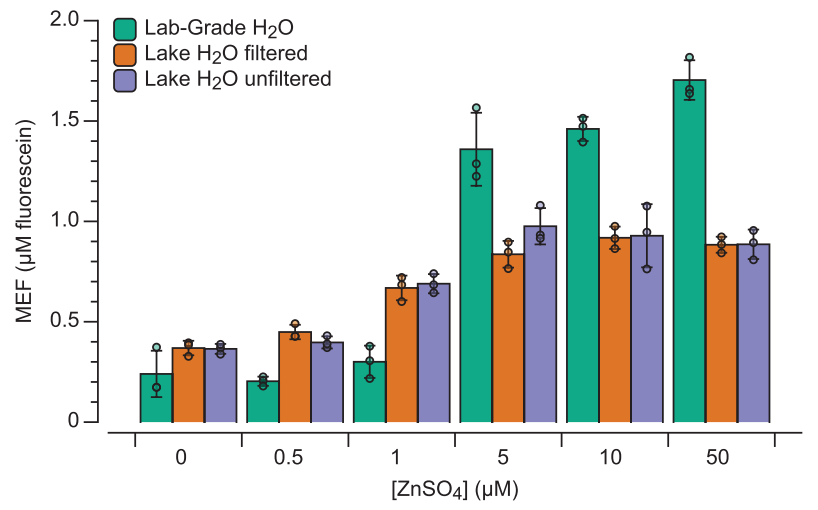

Extended Data Fig. 10 | Freeze-dried ROSALIND with TMSD can be rehydrated with real-world water matrices. Zinc-sensing ROSALIND with TMSD reactions that use smtO-InvadeR-InvadeR shown in Extended Data Fig. $4 \mathrm{~b}$ were freeze-dried and rehydrated with $\mathbf{a}$, tap water and $\mathbf{b}$, Lake Michigan water spiked with a range of concentrations of $\mathrm{ZnSO}_{4}$. The reactions were then incubated at $37^{\circ} \mathrm{C}$ for 1 hour and characterized for fluorescence. For each water sample type, the signal was compared to that of the reactions rehydrated with laboratory-grade water spiked with the same amount of $\mathrm{ZnSO}_{4}$. In each case, the reactions behaved as expected and saw no difference in signal between filtered and unfiltered water samples. However, a slight signal reduction is observed from the real-world water samples compared to the ones rehydrated with the $\mathrm{ZnSO}_{4}$ spiked laboratory-grade water likely due to matrix effects. All data shown are $n=3$ independent biological replicates each plotted as a point with raw fluorescence values standardized to MEF ( $\mu M$ fluorescein). Each bar height represents the average of the replicates, and error bars indicate the average of the replicates \pm standard deviation. 


\section{Reporting Summary}

Nature Portfolio wishes to improve the reproducibility of the work that we publish. This form provides structure for consistency and transparency in reporting. For further information on Nature Portfolio policies, see our Editorial Policies and the Editorial Policy Checklist.

\section{Statistics}

For all statistical analyses, confirm that the following items are present in the figure legend, table legend, main text, or Methods section.

$\mathrm{n} / \mathrm{a}$ Confirmed

$\bigotimes$ The exact sample size $(n)$ for each experimental group/condition, given as a discrete number and unit of measurement

$\square$ A statement on whether measurements were taken from distinct samples or whether the same sample was measured repeatedly

The statistical test(s) used AND whether they are one- or two-sided

Only common tests should be described solely by name; describe more complex techniques in the Methods section.

Х $\square$ A description of all covariates tested

Х $\square$ A description of any assumptions or corrections, such as tests of normality and adjustment for multiple comparisons

A full description of the statistical parameters including central tendency (e.g. means) or other basic estimates (e.g. regression coefficient)

AND variation (e.g. standard deviation) or associated estimates of uncertainty (e.g. confidence intervals)

For null hypothesis testing, the test statistic (e.g. $F, t, r$ ) with confidence intervals, effect sizes, degrees of freedom and $P$ value noted

Give $P$ values as exact values whenever suitable.

Х $\square$ For Bayesian analysis, information on the choice of priors and Markov chain Monte Carlo settings

Х $\square$ For hierarchical and complex designs, identification of the appropriate level for tests and full reporting of outcomes

Х $\square$ Estimates of effect sizes (e.g. Cohen's $d$, Pearson's $r$ ), indicating how they were calculated

our web collection on statistics for biologists contains articles on many of the points above.

\section{Software and code}

Policy information about availability of computer code
Data collection BioTek Gen5 v2.04 (microplate reader software)
BioRad Image Lab Touch Software 1.2.0.12 (ChemiDoc imaging system software)
Spicy.Integrate Package in Python 3.7.6
Github link for the ODE codes used in this manuscript: https://git.io/Jtlh1

$\begin{array}{ll}\text { Data analysis } & \text { Microsoft Excel v16.45 } \\ & \text { Fiji-ImageJ v2.1.0/1.53c }\end{array}$

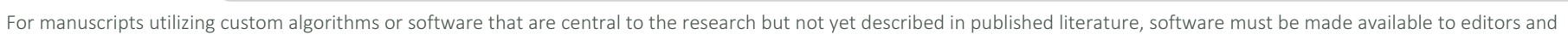

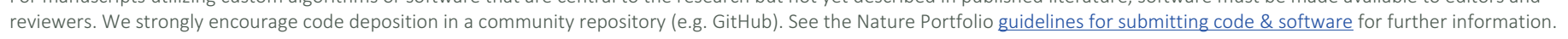

\section{Data}

Policy information about availability of data

All manuscripts must include a data availability statement. This statement should provide the following information, where applicable:

- Accession codes, unique identifiers, or web links for publicly available datasets

- A description of any restrictions on data availability

- For clinical datasets or third party data, please ensure that the statement adheres to our policy

All data presented in this manuscript are available as Source Data and as Supplementary Data. All Source Data as well as Supplementary Data 2 and 3 are also deposited in Mendeley Data (doi: 10.17632/hr3j3yztxb.1). All plasmids used in this manuscript are available in Addgene with the identifiers $140371,140374,140391$ and 140395. 
Please select the one below that is the best fit for your research. If you are not sure, read the appropriate sections before making your selection.

\section{Life sciences study design}

All studies must disclose on these points even when the disclosure is negative.

Sample size No sample size calculation was used. Sample size of $n=3$ was chosen for all quantitative fluorescence measurements based on similar cellfree / in vitro studies. While polyacrylamide gel electrophoresis experiments were performed three times, one representative of each analysis was included in the manuscript based on similar in vitro studies that involve PAGE analysis. All individual data points are shown in the figures, and total sample size spanning all independent experiments is reported in the figure captions.

Data exclusions No data on presented systems were excluded from the analyses. For the polyacrylamide gel images shown in Extended Data $1 \mathrm{~b}$, $\mathrm{f}$, we did test another condition, but did not discuss it in the manuscript as it was irrelevant to the claims made from the gel analysis. The uncropped, unprocessed gel images in Source Data as well as Supplementary Data 2 include this condition because all conditions tested were run alongside, but not discussed in the manuscript.

Replication All microplate reader experiments were performed three times by at least one co-author and are all presented in the manuscript. All polyacrylamide gel electrophoresis experiments were performed three times by at least one co-author, and one representatives of the replicates are presented in the manuscript. All attempts at replication were successful.

Randomization While randomization is not relevant to this study since our biochemical reactions are handled uniformly, the same data analysis procedure was applied to all samples of the same type.

Blinding Blinding is not relevant to this study since the results presented are based on objective description of our novel in vitro biosensing technology, and are therefore not subject to human biases.

\section{Reporting for specific materials, systems and methods}

We require information from authors about some types of materials, experimental systems and methods used in many studies. Here, indicate whether each material, system or method listed is relevant to your study. If you are not sure if a list item applies to your research, read the appropriate section before selecting a response.

\begin{tabular}{|c|c|c|c|}
\hline \multicolumn{2}{|r|}{ Materials \& experimental systems } & \multicolumn{2}{|c|}{ Methods } \\
\hline $\mathrm{n} / \mathrm{a}$ & Involved in the study & $\mathrm{n} / \mathrm{a}$ & Involved in the study \\
\hline Х & $\square$ Antibodies & Х & $\square$ ChIP-seq \\
\hline$\bigotimes$ & $\square$ Eukaryotic cell lines & Х & $\square$ Flow cytometry \\
\hline$\bigotimes$ & $\square$ Palaeontology and archaeology & Х & $\square$ MRI-based neuroimaging \\
\hline$\bigotimes$ & $\square$ Animals and other organisms & & \\
\hline Х & $\square$ Human research participants & & \\
\hline$凶$ & $\square$ Clinical data & & \\
\hline$\bigotimes$ & $\square$ Dual use research of concern & & \\
\hline
\end{tabular}

